\title{
A Systematic Review of the Association of Skipping Breakfast with Weight and Cardiometabolic Risk Factors in Children and Adolescents. What Should We Better Investigate in the Future?
}

\author{
Alice Monzani ${ }^{1,+}+$ (i) Roberta Ricotti ${ }^{1,+}$, Marina Caputo ${ }^{2}$, Arianna Solito ${ }^{1}$, Francesca Archero ${ }^{1}$, \\ Simonetta Bellone ${ }^{1,3}$ and Flavia Prodam 1,2,3,*(D) \\ 1 SCDU of Pediatrics, Department of Health Sciences, University of Piemonte Orientale, 28100 Novara, Italy; \\ alice.monzani@gmail.com (A.M.); roberta.ricotti@uniupo.it (R.R.); arisolito@gmail.com (A.S.); \\ francesca.archero@gmail.com (F.A.); simonetta.bellone@med.uniupo.it (S.B.) \\ 2 Endocrinology, Department of Translational Medicine, University of Piemonte Orientale, \\ 28100 Novara, Italy; marina.caputo@hotmail.com \\ 3 Interdisciplinary Research Center of Autoimmune Diseases, University of Piemonte Orientale, \\ 28100 Novara, Italy \\ * Correspondence: flavia.prodam@med.uniupo.it \\ + These authors contributed equally to this work.
}

Received: 30 December 2018; Accepted: 6 February 2019; Published: 13 February 2019

\begin{abstract}
The incidence of skipping breakfast in pediatric subjects is rising, and a relationship with overweight $(\mathrm{OW})$ and obesity $(\mathrm{OB})$ has been shown. Associations with cardiovascular outcomes and skipping breakfast in adults have been reported. The purpose of this systematic review was to summarize the association of skipping breakfast with body weight and metabolic outcomes in the pediatric population. We searched relevant databases (2008-2018) and identified 56 articles, of which 39 were suitable to be included, basing on inclusion criteria (observational; defined breakfast skipping; weight and/or metabolic outcomes). Overall, 286,804 children and adolescents living in 33 countries were included. The definitions of $\mathrm{OW} / \mathrm{OB}$, skipping breakfast, and the nutrient assessment were highly heterogeneous. Confounding factors were reported infrequently. The prevalence of skipping breakfast ranged 10-30\%, with an increasing trend in adolescents, mainly in girls. Skipping breakfast was associated with OW/OB in the $94.7 \%$ of the subjects. The lack of association was shown mainly in infants. Moreover, 16,130 subjects were investigated for cardiometabolic outcomes. Skipping breakfast was associated with a worse lipid profile, blood pressure levels, insulin-resistance, and metabolic syndrome. Five studies reported a lower quality dietary intake in breakfast skippers. This review supports skipping breakfast as an easy marker of the risk of OW/OB and metabolic diseases, whether or not it is directly involved in causality. We encourage intervention studies using standardized and generalizable indicators. Data on confounders, time of fasting, chronotypes, and nutrition quality are needed to establish the best practice for using it as a tool for assessing obesity risk.
\end{abstract}

Keywords: children; adolescents; breakfast; skipping; obesity; metabolic syndrome

\section{Introduction}

Childhood obesity $(\mathrm{OB})$ is a major public health issue in both developed and developing countries across the world [1]. Overweight (OW) and OB result from a complex network in which several factors interplay, such as genetic implications, birth weight, breastfeeding, parental obesity, physical 
activity, socioeconomic status, age, and gender [2]. Among them, dietary habits certainly also play a role [3]; in particular breakfast, the first meal in the day, has a critical role in energy balance and dietary regulation [4]. Despite this, the incidence of skipping breakfast among children and adolescent is rising $[4,5]$, and several studies have shown a positive relationship between breakfast skipping and OW/OB [6]. As a possible explanation, children who regularly have breakfast have been shown to be more likely to have a better diet quality and a higher intake of key food groups, such as fruit, dairy, and dietary fibers and, furthermore, they are also more likely to meet the recommendations for micronutrients [7-9]. Children who skip breakfast, instead, tend to eat more energy-dense food such as fast food leading to excess hunger and overeating [10].

The evidence on the association of breakfast consumption with body weight in the European population has been collected in the systematic review published in 2010; collectively, the data from observational studies carried out in Europe until 2009 have consistently demonstrated that children and adolescents who eat breakfast have a reduced risk of becoming overweight or obese and have a lower Body Mass Index (BMI) compared with those who skip breakfast [6]. Moreover, a series of studies have reported that breakfast skipping is associated with hypertension, cardiometabolic disease, insulin insensitivity, diabetes mellitus, and mortality [11]. However, these metabolic outcomes have not been explored in a larger systematic review and this associated has not been confirmed.

The aim of our systematic review is to analyze the association of skipping breakfast, methodologically defined based on reported questionnaires, with body weight and metabolic outcomes in the pediatric population, focusing on the studies published in the last ten years.

\section{Methods}

\subsection{Literature Search}

PICO methodology (Population: children and adolescents; Exposure: skipping breakfast; Comparison: not skipping breakfast; Outcomes; weight and metabolic parameters) to develop a search strategy based on medical subject headings (MeSH) and keywords were used. Guidelines of the Preferred Reporting for Systematic Reviews and Meta-Analyses (PRISMA) statement were followed, and a PRISMA checklist were followed.

The Cochrane Central Register of Controlled Trials, PubMed, CINHAHL, and EMBASE databases (January 2008-December 2018) were systematically used. The reference lists of identified studies and key review articles, including previously published reviews, were also searched for all randomized and non-randomized clinical trials as well as prospective cohort and cross-sectional studies assessing the association of breakfast skipping or consumption, however, defined, with body weight and cardiometabolic aspects in children and adolescents. No country restrictions were imposed.

The search terms used included "breakfast", and "children\$" (or "adolescents\$"). The search strategy used both keywords and MeSH terms. No further limitations were made so the search terms would be as sensitive as possible. In addition, we checked the references of eligible articles for further papers that were not captured by our search strategy and we corresponded with authors when the relevant information was missing in the paper.

\subsection{Outcome Measures}

The primary outcome measures were as follows: body weight, body weight excess (e.g., overweight, obesity), and body mass index (BMI); for cardio-metabolic aspects we considered metabolic syndrome (MetS), arterial hypertension, lipid profile, glucose levels, type 2 diabetes, insulin resistance, and uric acid. Included studies had to report at least one of these primary outcomes. 


\subsection{Inclusion and Exclusion Criteria}

For inclusion, studies were required to (i) include children and/or adolescents aged 2-18 years (or a mean within these ranges) as subjects of study. Studies that did not state the mean age of participants were classified as child or adolescent studies depending on the ages of the majority of the sample; (ii) have a defined measure of the child's or adolescent's breakfast consumption and/or breakfast skipping; (iii) be published in peer-reviewed journals in the English language; (vii) be published in the period January 2008-December 2018. We included studies even if they did not report completely the dietary quality data. We excluded intervention studies, studies where overweight/obese subjects were the only participants, and studies focusing on eating disorders.

\subsection{Identification of Relevant Studies}

Potentially relevant papers were selected by reading the titles and abstracts. If abstracts were not available or did not provide enough results the entire article was retrieved and screened to determine whether it met the inclusion criteria.

\subsection{Data Extraction, Synthesis, and Quality Assessment}

A form was generated to register whether individual studies met eligibility criteria and to collect data regarding the study design and methodological quality. Three investigators independently reviewed and extracted data from the papers according to the predetermined criteria. Any difference in opinion about the studies was resolved by discussion between all the investigators. The following data were extracted: author, date of publication, study design, characteristics of the participants (sample size, age, gender, and country), measures of breakfast behaviors, breakfast behavior (i.e., breakfast consumption/skipping), assessment methodology and reliability and validity of dietary measures, definition of weight excess and assessment methodology. This information is summarized in Table 1. About secondary outcomes also the following data were extracted: definition of Mets, blood pressure, lipid profile, glucose, insulin, and uric acid levels, indexes of insulin resistance. Parameters could be as continuous or dichotomous variables. If reported, data on nutrient intake quality were included. This information is summarized in Table 2. 
Table 1. Summarized studies' characteristics on weight and skipping breakfast.

\begin{tabular}{|c|c|c|c|c|c|c|c|c|}
\hline $\begin{array}{l}\text { Reference } \\
\text { (Author, } \\
\text { year, } n \text { ) }\end{array}$ & Study Subjects & Methods & $\begin{array}{l}\text { Skipping Breakfast } \\
\text { Definition }\end{array}$ & $\begin{array}{l}\text { Breakfast Evaluation } \\
\text { Method }\end{array}$ & OB/OW Definition & $\begin{array}{l}\text { Prevalence of } \\
\text { Breakfast Skippers }\end{array}$ & Results & $\begin{array}{l}\text { Impact of } \\
\text { Skipping } \\
\text { Breakfast } \\
\text { on OW/OB }\end{array}$ \\
\hline $\begin{array}{l}\text { Dialektakou } \\
2008 \text { [12] }\end{array}$ & $\begin{array}{l}\mathrm{N}=811, \text { aged } \\
14.9-21.2 \text { years, } \\
\mathrm{M} \text { and } \mathrm{F} \text {, Greece }\end{array}$ & $\begin{array}{l}\text { Cross-sectional, self-reported } \\
\text { questionnaires, measured } \\
\text { height and weight }\end{array}$ & $\begin{array}{l}24 \text { definitions evaluating } \\
\text { breakfast consumption on } \\
\text { the day of data collection, } \\
\text { during the whole year, } \\
\text { during the previous week, } \\
\text { and on weekends/holidays }\end{array}$ & Not specified & $\begin{array}{l}\text { Age- and } \\
\text { sex-specific BMI } \\
\text { cut-offs according } \\
\text { to IOTF criteria }\end{array}$ & $\begin{array}{l}3.6-74.7 \% \\
\text { (according to } \\
\text { different } \\
\text { definitions) }\end{array}$ & $\begin{array}{l}\text { Fewer breakfast-skipping } \\
\text { variables associated with BMI than } \\
\text { with OW/OB. Fewer associations } \\
\text { when controlling for potential } \\
\text { confounders. Fewer associations } \\
\text { for variables corresponding to } \\
\text { some definitions. }\end{array}$ & $\uparrow$ \\
\hline $\begin{array}{l}\text { Dubois } 2008 \\
\text { [13] }\end{array}$ & $\begin{array}{l}\mathrm{N}=1549 ; \text { aged } \\
44-56 \text { months, } \\
\text { M and F, } \\
\text { Canada }\end{array}$ & $\begin{array}{l}\text { Longitudinal study, } \\
\text { parent-report questionnaires, } \\
\text { measured height and weight } \\
\text { (cross-sectional data } \\
\text { considered) }\end{array}$ & $\begin{array}{l}\text { Frequency of breakfast } \\
\text { eating: (1) yes, every } \\
\text { morning; (2) regularly but } \\
\text { not every day; (3) only on } \\
\text { occasion; and (4) never. } \\
\text { Categories } 2 \text { to } 4 \text { were } \\
\text { classified as 'breakfast } \\
\text { skippers' }\end{array}$ & $\begin{array}{l}\text { Eating behavior } \\
\text { questionnaire (Enquete } \\
\text { sociale et de sante' aupres } \\
\text { des enfants et des } \\
\text { adolescents Quebecois } \\
\text {-Health and Social Survey } \\
\text { of Quebec Children and } \\
\text { Adolescents) and a } 24 \mathrm{~h} \\
\text { dietary recall interview }\end{array}$ & $\begin{array}{l}\text { Age- and } \\
\text { sex-specific BMI } \\
\text { z-score cut-offs } \\
\text { according to CDC } \\
\text { criteria }\end{array}$ & $10 \%$ & $\begin{array}{l}\uparrow \text { intake of energy, carbohydrates } \\
\text { or servings of grain products in } \\
\text { breakfast skippers }\end{array}$ & $\uparrow$ \\
\hline $\begin{array}{l}\text { Harding } \\
2008 \text { [14] }\end{array}$ & $\begin{array}{l}\mathrm{N}=6599, \text { aged } \\
11-13 \text { years, } \mathrm{M} \\
\text { and } \mathrm{F}, \mathrm{UK}\end{array}$ & $\begin{array}{l}\text { Cross-sectional, self-reported } \\
\text { questionnaires, measured } \\
\text { height and weight }\end{array}$ & $\begin{array}{l}\text { Number of eaten breakfasts } \\
\text { per week (Every day; } \\
\text { 3-4 days a week; } 1-2 \text { days a } \\
\text { week; Never or hardly ever) }\end{array}$ & $\begin{array}{l}\text { http://www.sphsu.mrc. } \\
\text { ac.uk/studies/dash/ } \\
\text { Food frequency } \\
\text { questionnaire }\end{array}$ & $\begin{array}{l}\text { Age- and } \\
\text { sex-specific BMI } \\
\text { cut-offs according } \\
\text { to IOTF criteria }\end{array}$ & $\begin{array}{l}32.6-53 \% \text { not eating } \\
\text { breakfast every day }\end{array}$ & $\begin{array}{l}\text { Increased risk for obesity in } \\
\text { breakfast skippers (girls OR 1.74, } \\
95 \% \text { CI 1.30-2.34; boys OR 2.06; CI } \\
\text { 1.57-2.70) }\end{array}$ & $\uparrow$ \\
\hline $\begin{array}{l}\text { Duncan } 2008 \\
\text { [15] }\end{array}$ & $\begin{array}{l}\mathrm{N}=1229, \text { aged } \\
5-11 \text { years, } \mathrm{M} \\
\text { and F, New } \\
\text { Zeland }\end{array}$ & $\begin{array}{l}\text { Cross-sectional, proxy } \\
\text { questionnaire administered } \\
\text { to the parents, measured } \\
\text { height and weight, BIA }\end{array}$ & $\begin{array}{l}\text { Number of eaten breakfasts } \\
\text { in the last full week } \\
\text { (0-7 days per week) }\end{array}$ & 7-day recall & $\begin{array}{l}\text { Boys and girls were } \\
\text { classified as } \\
\text { "overfat" if their \% } \\
\text { BF exceeded } 25 \% \\
\text { and 30\% } \\
\text { (respectively) }\end{array}$ & $\begin{array}{l}1.3 \\
\text { (non-overfat)- } 2.6 \% \\
\text { (overfat) never eat } \\
\text { breakfast in a week }\end{array}$ & $\begin{array}{l}\text { Breakfast skippers had increased } \\
\text { odds of overfat compared with } \\
\text { those who had breakfast for five or } \\
\text { more days/week. }\end{array}$ & $\uparrow$ \\
\hline $\begin{array}{l}\text { Croezen } \\
2009[16]\end{array}$ & $\begin{array}{l}\mathrm{N}=25176 \text {, aged } \\
13-16 \text { years, } \mathrm{M} \\
\text { and F, The } \\
\text { Netherlands }\end{array}$ & $\begin{array}{l}\text { Cross-sectional, detailed } \\
\text { Internet questionnaire, under } \\
\text { supervision of instructed } \\
\text { teachers following a } \\
\text { standardized protocol, } \\
\text { self-reported body weight } \\
\text { and height }\end{array}$ & $\begin{array}{l}\text { Number of eaten breakfasts } \\
\text { per week (0-7 days per } \\
\text { week) }\end{array}$ & $\begin{array}{l}\text { Food frequency } \\
\text { questionnaire }\end{array}$ & $\begin{array}{l}\text { Age- and } \\
\text { sex-specific BMI } \\
\text { cut-offs according } \\
\text { to Cole's definition }\end{array}$ & $29.3-39.2 \%$ & $\begin{array}{l}\text { Skipping breakfast }>2 \text { times/week } \\
\text { associated with OW (adjusted OR } \\
1.68 \text { (CI 1.43-1.97) in 13-14 } \\
\text { year-aged, and } 1.32 \text { (CI 1.14-1.54) } \\
\text { in 15-16 year-aged subjects); } \\
\text { skipping breakfast every day } \\
\text { associated with OB }\end{array}$ & $\uparrow$ \\
\hline $\begin{array}{l}\text { Nagel } 2009 \\
{[17]}\end{array}$ & $\begin{array}{l}\mathrm{N}=1079 \text {, aged } \\
6.2-9.2 \text { years, } \mathrm{M} \\
\text { and } \mathrm{F} \text {, Germany }\end{array}$ & $\begin{array}{l}\text { Cross-sectional, self-reported } \\
\text { questionnaires compiled by } \\
\text { children and parents, } \\
\text { measured weight, height, } \\
\text { upper arm and waist } \\
\text { circumference, skin fold } \\
\text { thickness }\end{array}$ & $\begin{array}{l}\text { Breakfast consumption } \\
\text { before school (yes/no) }\end{array}$ & Not specified & $\begin{array}{l}\text { Age- and } \\
\text { sex-specific BMI } \\
\text { cut-offs according } \\
\text { to IOTF criteria }\end{array}$ & $13.4 \%$ & $\begin{array}{l}\text { Breakfast skippers had increased } \\
\text { risk for OW (OR 1.73, } 95 \% \text { CI } \\
1.13-2.64) \text { and OB (OR 2.50, } \\
95 \% \text { CI 1.19-5.29) }\end{array}$ & $\uparrow$ \\
\hline
\end{tabular}


Table 1. Cont.

\begin{tabular}{|c|c|c|c|c|c|c|c|c|}
\hline $\begin{array}{l}\text { Reference } \\
\text { (Author, } \\
\text { year, } n \text { ) }\end{array}$ & Study Subjects & Methods & $\begin{array}{l}\text { Skipping Breakfast } \\
\text { Definition }\end{array}$ & $\begin{array}{l}\text { Breakfast Evaluation } \\
\text { Method }\end{array}$ & OB/OW Definition & $\begin{array}{l}\text { Prevalence of } \\
\text { Breakfast Skippers }\end{array}$ & Results & $\begin{array}{l}\text { Impact of } \\
\text { Skipping } \\
\text { Breakfast } \\
\text { on OW/OB }\end{array}$ \\
\hline Sun 2009 [18] & $\begin{array}{l}N=5753 \text {, aged } \\
12-13 \text { years, } M \\
\text { and } F \text {, Japan }\end{array}$ & $\begin{array}{l}\text { Cross-sectional, self-reported } \\
\text { questionnaires, measured } \\
\text { height and weight }\end{array}$ & $\begin{array}{l}\text { Frequency of eating } \\
\text { breakfast: daily, almost } \\
\text { daily, sometimes, and rarely }\end{array}$ & $\begin{array}{l}\text { Food frequency } \\
\text { questionnaire }\end{array}$ & $\begin{array}{l}\text { Age- and } \\
\text { sex-specific BMI } \\
\text { cut-offs according } \\
\text { to IOTF criteria }\end{array}$ & $\begin{array}{l}1.1 \% \text { of boys and } \\
0.7 \% \text { of girls ate } \\
\text { breakfast rarely }\end{array}$ & $\begin{array}{l}\text { Skipping breakfast (i.e., eating } \\
\text { breakfast rarely) was associated } \\
\text { with OW (in boys only after } \\
\text { adjustment for age, parental OW } \\
\text { and lifestyle variables) }\end{array}$ & $\uparrow$ \\
\hline $\begin{array}{l}\text { Maddah } \\
2010[19]\end{array}$ & $\begin{array}{l}\mathrm{N}=6635, \text { aged } \\
6-11 \text { years, } \mathrm{M} \\
\text { and } \mathrm{F} \text {, Iran }\end{array}$ & $\begin{array}{l}\text { Cross-sectional, self-reported } \\
\text { questionnaire given to the } \\
\text { parents, measured weight } \\
\text { and height }\end{array}$ & Breakfast skipping (yes/no) & Not specified & $\begin{array}{l}\text { Age- and } \\
\text { sex-specific BMI } \\
\text { cut-offs according } \\
\text { to IOTF criteria }\end{array}$ & Not reported & $\begin{array}{l}\text { Higher prevalence of OW/OB in } \\
\text { breakfast skippers than in } \\
\text { breakfast eaters (boys: } 23.6 \% \\
\text { versus } 16.9 \% \text {, girls: } 23.5 \% \\
\text { versus } 17.1 \% \text { ) }\end{array}$ & $\uparrow$ \\
\hline $\begin{array}{l}\text { Isacco } 2010 \\
\text { [20] }\end{array}$ & $\begin{array}{l}\mathrm{N}=278, \text { aged } \\
6-10 \text { years, } \mathrm{M} \\
\text { and F, France }\end{array}$ & $\begin{array}{l}\text { Cross-sectional, self-reported } \\
\text { questionnaire compiled by } \\
\text { the parents in the presence of } \\
\text { their child, measured weight, } \\
\text { height, WC and skin fold } \\
\text { thickness }\end{array}$ & $\begin{array}{l}\text { Frequency of eating } \\
\text { breakfast: every day, } \\
\text { sometimes, never }\end{array}$ & $\begin{array}{l}\text { Food frequency } \\
\text { questionnaire }\end{array}$ & $\begin{array}{l}\text { Age- and } \\
\text { sex-specific BMI } \\
\text { z-score cut-offs } \\
\text { according to } \\
\text { CDC criteria }\end{array}$ & $\begin{array}{l}1.4 \% \text { never ate } \\
\text { breakfast }\end{array}$ & $\begin{array}{l}\text { higher BMI } z \text {-score, skinfolds and } \\
\text { WC in breakfast skippers }\end{array}$ & $\uparrow$ \\
\hline $\begin{array}{l}\text { Deshmukh-Tas } \\
2010 \text { [21] }\end{array}$ & $\begin{array}{l}\mathrm{N}=9659 \text {, aged } \\
\mathrm{kg}-18 \text { years, M } \\
\text { and F, USA }\end{array}$ & $\begin{array}{l}\text { Cross-sectional, self-reported } \\
\text { data on 24-h recall } \\
\text { methodology over two days } \\
\text { (assisted by } \\
\text { parent/caregivers for } \\
\text { children aged } 6 \text { to } 11 \text { years), } \\
\text { measured weight, height } \\
\text { and WC }\end{array}$ & $\begin{array}{l}\text { Breakfast skippers: those } \\
\text { who consumed no food or } \\
\text { beverages, excluding water, } \\
\text { at breakfast }\end{array}$ & $\begin{array}{l}\text { 24-h recall } \\
\text { http: } \\
\text { //www.cdc.gov/nchs/ } \\
\text { data/nhanes/dr-1-5.pdf. }\end{array}$ & $\begin{array}{l}\text { Age- and } \\
\text { sex-specific BMI } \\
\text { z-score cut-offs } \\
\text { according to } \\
\text { CDC criteria }\end{array}$ & $\begin{array}{l}20 \% \text { of children, } \\
31.5 \% \text { of } \\
\text { adolescents }\end{array}$ & $\begin{array}{l}\text { Breakfast skippers had higher BMI } \\
z \text {-scores and a higher waist } \\
\text { circumference than ready-to-eat } \\
\text { cereal and other breakfast } \\
\text { consumers. Higher prevalence of } \\
\text { obesity in breakfast skippers than } \\
\text { ready-to-eat cereal consumers }\end{array}$ & $\uparrow$ \\
\hline So 2011 [22] & $\begin{array}{l}\mathrm{N}=11570, \text { aged } \\
9-18 \text { years, } \mathrm{M} \\
\text { and } \mathrm{F}, \\
\text { Hong Kong }\end{array}$ & $\begin{array}{l}\text { Cross-sectional, self-reported } \\
\text { questionnaires, measured } \\
\text { height and weight, and BIA }\end{array}$ & $\begin{array}{l}\text { Breakfast skippers (ate } \\
\text { breakfast 0-2 times/week); } \\
\text { semi-skippers (ate breakfast } \\
\text { 3-4 times/week); } \\
\text { non-skippers (ate breakfast } \\
\text { 5-7 times/week) }\end{array}$ & $\begin{array}{l}\text { Rapid Dietary behavior } \\
\text { Assessment questionnaire } \\
\text { (daily and weekly dietary } \\
\text { behaviors, validated } \\
\text { against the } 24 \mathrm{~h} \text { recall } \\
\text { nutrient intake data in a } \\
\text { smaller sample) }\end{array}$ & $\begin{array}{l}\text { Age- and } \\
\text { sex-specific BMI } \\
\text { cut-offs according } \\
\text { to IOTF criteria }\end{array}$ & $\begin{array}{l}8 \% \text { of primary } \\
\text { school students and } \\
14 \% \text { of secondary } \\
\text { school students }\end{array}$ & $\begin{array}{l}\text { Breakfast skippers had higher BMI, } \\
\text { BMI } z \text {-scores and BF\% than their } \\
\text { counterparts }\end{array}$ & $\uparrow$ \\
\hline Tin 2011 [23] & $\begin{array}{l}\mathrm{N}=113457, \\
\text { aged } 9-10 \text { years, } \\
\mathrm{M} \text { and } \mathrm{F}, \\
\text { Hong Kong }\end{array}$ & $\begin{array}{l}\text { Longitudinal, 2-year } \\
\text { follow-up, self-reported } \\
\text { questionnaires, measured } \\
\text { height and weight } \\
\text { (cross-sectional data } \\
\text { considered) }\end{array}$ & $\begin{array}{l}\text { Breakfast skippers those } \\
\text { who chose 'no breakfast } \\
\text { at all' }\end{array}$ & Not specified & $\begin{array}{l}\text { Age- and } \\
\text { sex-specific BMI } \\
\text { cut-offs according } \\
\text { to IOTF criteria }\end{array}$ & $\begin{array}{l}5.3 \% \text { of boys, } 5.2 \% \\
\text { of girls }\end{array}$ & $\begin{array}{l}\text { Higher mean BMI in breakfast } \\
\text { skippers both at baseline ( } \beta 0.77 \text {, } \\
95 \% \text { CI } 0.67-0.87) \text { and } 2 \text { years later } \\
(\beta 0.86,95 \% \text { CI } 0.78-0.95)\end{array}$ & $\uparrow$ \\
\hline
\end{tabular}


Table 1. Cont.

\begin{tabular}{|c|c|c|c|c|c|c|c|c|}
\hline $\begin{array}{l}\text { Reference } \\
\text { (Author, } \\
\text { year, } n \text { ) }\end{array}$ & Study Subjects & Methods & $\begin{array}{l}\text { Skipping Breakfast } \\
\text { Definition }\end{array}$ & $\begin{array}{l}\text { Breakfast Evaluation } \\
\text { Method }\end{array}$ & OB/OW Definition & $\begin{array}{l}\text { Prevalence of } \\
\text { Breakfast Skippers }\end{array}$ & Results & $\begin{array}{l}\text { Impact of } \\
\text { Skipping } \\
\text { Breakfast } \\
\text { on OW/OB }\end{array}$ \\
\hline $\begin{array}{l}\text { Mushtaq } \\
2011[24]\end{array}$ & $\begin{array}{l}N=1860, \text { aged } \\
5-12 \text { years, M } \\
\text { and F, Pakistan }\end{array}$ & $\begin{array}{l}\text { Cross-sectional, } \\
\text { questionnaires administered } \\
\text { to the children by senior } \\
\text { medical students, measured } \\
\text { height and weight }\end{array}$ & $\begin{array}{l}\text { Skipping breakfast once or } \\
\text { more in the past week }\end{array}$ & 7-day recall & $\begin{array}{l}\text { BMI } z \text {-scores } \\
\text { calculated based on } \\
\text { the WHO criteria }\end{array}$ & $8 \%$ & $\begin{array}{l}\text { Breakfast skippers were } \\
\text { significantly more likely to be } \\
\text { overweight }(15 \% \text { versus } 9 \% \text { ) and } \\
\text { obese ( } 13 \% \text { versus } 7 \% \text { ) than } \\
\text { breakfast eaters }(p=0.002) \text {. } \\
\text { Skipping breakfast was associated } \\
\text { with overweight among girls } \\
(p<0.001) \text {. Skipping breakfast as } \\
\text { independent predictor of OW (OR } \\
1.82,95 \% \text { CI 1.22-2.71) }\end{array}$ & $\begin{array}{l}\uparrow(\mathrm{OW} \text { in } \\
\text { girls })\end{array}$ \\
\hline $\begin{array}{l}\text { Kuriyan } \\
2012[25]\end{array}$ & $\begin{array}{l}\mathrm{N}=8444 \\
(4707 \text { aged } \\
3-10 \text { years; } \\
\mathrm{N}=3737 \text { aged } \\
10-16 \text { years), } \mathrm{M} \\
\text { and } \mathrm{F} \text {, Bangalore }\end{array}$ & $\begin{array}{l}\text { Cross-sectional, } \\
\text { parent/student-report } \\
\text { questionnaires, measured } \\
\text { height and weight, WC }\end{array}$ & Breakfast skipping (yes/no) & Not specified & $\begin{array}{l}\text { Indian Academy of } \\
\text { Pediatrics cut-off } \\
\text { for BMI; WC }>75 \text { th } \\
\text { percentile for } \\
\text { classifying } \\
\text { abdominal obesity }\end{array}$ & Not reported & - & $\begin{array}{l}\Leftrightarrow \text { WC in } \\
\text { children } \\
\text { aged } \\
3-10 \text { years } \uparrow \\
\text { WC in } \\
\text { children } \\
\text { aged } 10-16 \\
\text { years }\end{array}$ \\
\hline $\begin{array}{l}\text { Kyeariazis } \\
2012[26]\end{array}$ & $\begin{array}{l}\mathrm{N}=2374, \text { aged } \\
6-12 \text { years, } \mathrm{M} \\
\text { and F, Greece }\end{array}$ & $\begin{array}{l}\text { Cross-sectional, self-reported } \\
\text { questionnaires, measured } \\
\text { height and weight }\end{array}$ & Breakfast skipping (yes/no) & $\begin{array}{l}\text { Closed format questions } \\
\text { in the form of multiple } \\
\text { choice Questions }\end{array}$ & $\begin{array}{l}\text { Age- and } \\
\text { sex-specific BMI } \\
\text { cut-offs according } \\
\text { to Cole's definition }\end{array}$ & Not reported & $\begin{array}{l}\text { Skipping breakfast had a positive } \\
\text { association with OB }\end{array}$ & $\uparrow$ \\
\hline $\begin{array}{l}\text { Van } \\
\text { Lippevelde } \\
2013 \text { [27] }\end{array}$ & $\begin{array}{l}\mathrm{N}=6374, \text { aged } \\
10-12 \text { years, M } \\
\text { and F, Belgium, } \\
\text { Greece, } \\
\text { Hungary, the } \\
\text { Netherlands, } \\
\text { Norway, } \\
\text { Slovenia, Spain, } \\
\text { and Switzerland }\end{array}$ & $\begin{array}{l}\text { Cross-sectional, self-reported } \\
\text { questionnaires compiled by } \\
\text { the children during } \\
\text { school-time, measured } \\
\text { weight and height }\end{array}$ & $\begin{array}{l}\text { Breakfast frequency per } \\
\text { week }(0-7) \text { calculated by } \\
\text { adding up the breakfasts } \\
\text { usually had on schooldays } \\
\text { per week }(0-5) \text { and on } \\
\text { weekend days per week } \\
(0-2)\end{array}$ & $\begin{array}{l}\text { http:/ / projectenergy.eu } \\
\text { Food frequency } \\
\text { questionnaire }\end{array}$ & $\begin{array}{l}\text { BMI } z \text {-scores } \\
\text { calculated based on } \\
\text { the WHO criteria }\end{array}$ & Not reported & $\begin{array}{l}\text { Children's breakfast consumption } \\
\text { negatively related to children's } \\
\text { BMI-z-score }\end{array}$ & $\uparrow$ \\
\hline $\begin{array}{l}\text { Januszek- } \\
\text { Trzciakowska } \\
2014 \text { [28] }\end{array}$ & $\begin{array}{l}\mathrm{N}=2571, \text { aged } \\
7-9 \text { years, M } \\
\text { and F, Poland }\end{array}$ & $\begin{array}{l}\text { Cross-sectional, self-reported } \\
\text { questionnaire compiled by } \\
\text { the parents, measured } \\
\text { weight and height }\end{array}$ & $\begin{array}{l}\text { Breakfast frequency: } \\
\text { always, usually never }\end{array}$ & $\begin{array}{l}\text { Food frequency } \\
\text { questionnaire }\end{array}$ & $\begin{array}{l}\text { Age- and } \\
\text { sex-specific BMI } \\
\text { cut-offs according } \\
\text { to IOTF criteria }\end{array}$ & $\begin{array}{l}10.3 \% \text { in girls, } 9.1 \% \\
\text { in boys }\end{array}$ & $\begin{array}{l}\text { Increased OB risk in girls } \\
\text { irregularly or never eating } \\
\text { breakfast (always versus usually, } \\
\text { OR } 2.71,95 \% \text { CI } 1.33-5.51 \text { always } \\
\text { versus never OR } 1.63,95 \% \text { CI } \\
1.08-2.47 \text { ) }\end{array}$ & $\begin{array}{l}\uparrow \text { only for } \\
\text { girls }\end{array}$ \\
\hline
\end{tabular}


Table 1. Cont.

\begin{tabular}{|c|c|c|c|c|c|c|c|c|}
\hline $\begin{array}{l}\text { Reference } \\
\text { (Author, } \\
\text { year, } n \text { ) }\end{array}$ & Study Subjects & Methods & $\begin{array}{l}\text { Skipping Breakfast } \\
\text { Definition }\end{array}$ & $\begin{array}{l}\text { Breakfast Evaluation } \\
\text { Method }\end{array}$ & OB/OW Definition & $\begin{array}{l}\text { Prevalence of } \\
\text { Breakfast Skippers }\end{array}$ & Results & $\begin{array}{l}\text { Impact of } \\
\text { Skipping } \\
\text { Breakfast } \\
\text { on OW/OB }\end{array}$ \\
\hline $\begin{array}{l}\text { Kupers } 2014 \\
\text { [29] }\end{array}$ & $\begin{array}{l}\text { T1: } 2 \text { years of } \\
\text { age; } N=1488 \\
\text { T2: } 5 \text { years of } \\
\text { age; } N=1366 \mathrm{M} \\
\text { and F, The } \\
\text { Netherlands }\end{array}$ & $\begin{array}{l}\text { Longitudinal; parent-report } \\
\text { questionnaires; measured } \\
\text { height and weight } \\
\text { (cross-sectional data } \\
\text { considered) }\end{array}$ & $\begin{array}{l}\text { Breakfast frequency per } \\
\text { week (0-7), categorized as } \\
\text { "eating breakfast daily" ( } \\
\text { times per week) or "not } \\
\text { eating breakfast daily" ( }<7 \\
\text { times per week) }\end{array}$ & $\begin{array}{l}\text { Food frequency } \\
\text { questionnaire }\end{array}$ & $\begin{array}{l}\text { Age- and } \\
\text { sex-specific BMI } \\
\text { cut-offs according } \\
\text { Dutch reference } \\
\text { growth charts }\end{array}$ & $\begin{array}{l}\text { At } \mathrm{T} 1,3.0 \% \text { of the } \\
\text { children did not eat } \\
\text { breakfast daily; at } \\
\text { T2, } 5.3 \%\end{array}$ & $\begin{array}{l}\text { No association between skipping } \\
\text { breakfast and overweight, neither } \\
\text { at age } 2 \text { nor at age } 5\end{array}$ & $\Leftrightarrow$ \\
\hline $\begin{array}{l}\text { O'Neil } 2015 \\
\text { [30] }\end{array}$ & $\begin{array}{l}\mathrm{N}=14200, \text { aged } \\
2-18 \text { years, } \mathrm{M} \\
\text { and F, USA }\end{array}$ & $\begin{array}{l}\text { Cross-sectional, self-reported } \\
\text { questionnaires (complied by } \\
\text { parents/guardians of 2-5 } \\
\text { year children; by 6-11 year } \\
\text { children assisted by an adult; } \\
\text { older children provided their } \\
\text { own recall), measured } \\
\text { weight and height }\end{array}$ & $\begin{array}{l}\text { 24-h dietary recall: no } \\
\text { breakfast or } 11 \text { possible } \\
\text { breakfast patterns }\end{array}$ & $\begin{array}{l}\text { 24-h dietary recall } \\
\text { interviews using an } \\
\text { automated multiple-pass } \\
\text { method } \\
\text { http://www.cdc.gov/ } \\
\text { nchs/data/nhanes/ } \\
\text { nhanes_03_04/ } \\
\text { DIETARY_MEC.pdf. }\end{array}$ & $\begin{array}{l}\text { Age- and } \\
\text { sex-specific BMI } \\
\text { cut-offs according } \\
\text { to CDC criteria }\end{array}$ & $18.7 \%$ & $\begin{array}{l}\text { Mean BMI z-scores were lower } \\
\text { among consumers of five breakfast } \\
\text { patterns (grain/lower fat } \\
\text { milk/sweets/fruit juice, } \\
\text { pre-sweetened ready-to-eat } \\
\text { cereal/whole milk, soft } \\
\text { drinks/fruit juice/grain/potatoes, } \\
\text { ready-to-eat cereal/whole milk, } \\
\text { and cooked cereal/milk/fruit } \\
\text { juice), compared to breakfast } \\
\text { skippers. }\end{array}$ & $\uparrow$ \\
\hline $\begin{array}{l}\text { Smetanina } \\
2015[31]\end{array}$ & $\begin{array}{l}\mathrm{N}=3990, \text { aged } \\
7-17 \text { years, } \mathrm{M} \\
\text { and } \mathrm{F}, \text { Lithuania }\end{array}$ & $\begin{array}{l}\text { Cross-sectional, self-reported } \\
\text { questionnaires (parents of } \\
\text { younger age ( } 7-9 \text { years old) } \\
\text { participants filled-in the } \\
\text { questionnaire at home and } \\
\text { older children and } \\
\text { adolescents filled-in it } \\
\text { themselves at school), } \\
\text { measured weight and height }\end{array}$ & $\begin{array}{l}\text { breakfast eating frequency } \\
\text { per week: "Everyday" } \\
(\text { "Everyday" and "4-6 times } \\
\text { per week"), "-3 times per } \\
\text { week", and "Never" }\end{array}$ & $\begin{array}{l}\text { Modified WHO } \\
\text { questionnaires } \\
\text { (conducted by Health } \\
\text { behavior in School-aged } \\
\text { Children (HBSC) and } \\
\text { COSI study groups).Food } \\
\text { frequency questionnaire }\end{array}$ & $\begin{array}{l}\text { Age- and } \\
\text { sex-specific BMI } \\
\text { cut-offs according } \\
\text { to IOTF criteria }\end{array}$ & $\begin{array}{l}\text { Never eating } \\
\text { breakfast: } 6.2 \% \text { in } \\
\text { underweight, } 6.5 \% \\
\text { in NW, } 9.6 \% \text { in } \\
\text { OW/OB }\end{array}$ & $\begin{array}{l}\text { The prevalence of subjects never } \\
\text { having breakfast was significantly } \\
\text { higher in OW/OB than in NW } \\
(9.6 \% \text { versus } 6.5 \%)\end{array}$ & $\uparrow$ \\
\hline $\begin{array}{l}\text { Zakrzewski } \\
2015 \text { [32] }\end{array}$ & $\begin{array}{l}\mathrm{N}=6841, \text { aged } \\
\text { 9-11 years, M } \\
\text { and F, Australia, } \\
\text { Brasil, Canada, } \\
\text { China, } \\
\text { Colombia, } \\
\text { Finland, India, } \\
\text { Kenya, Portugal, } \\
\text { South Africa, } \\
\text { UK, US }\end{array}$ & $\begin{array}{l}\text { Cross-sectional, self-reported } \\
\text { questionnaires, measured } \\
\text { height, weight and } \mathrm{BF} \%\end{array}$ & $\begin{array}{l}\text { Breakfast frequency per } \\
\text { week (separately for } \\
\text { weekdays and weekend } \\
\text { days). } 1 \text {. Three-category } \\
\text { definition: weekly breakfast } \\
\text { frequency coded as rare } \\
\text { (0-2 days per week), } \\
\text { occasional (3-5 days per } \\
\text { week) and frequent } \\
\text { (6-7 days per week). } 2 \text {. } \\
\text { Two-category definition: } \\
\text { weekly breakfast frequency } \\
\text { recoded as less than daily } \\
\text { (0-6 days per week) or daily } \\
\text { ( } 7 \text { days per week). }\end{array}$ & $\begin{array}{l}\text { Food frequency } \\
\text { questionnaire }\end{array}$ & $\begin{array}{l}\text { BMI } z \text {-scores } \\
\text { calculated based on } \\
\text { the WHO criteria }\end{array}$ & $\begin{array}{l}\text { Breakfast } \\
\text { consumption: } 6.3 \% \\
\text { rarely, } 27.7 \% \text { less } \\
\text { than daily }\end{array}$ & $\begin{array}{l}\text { Frequent breakfast consumption } \\
\text { was associated with lower BMI } \\
\text { z-scores compared with occasional } \\
(p<0.0001) \text { and rare }(p<0.0001) \\
\text { consumption, as well as lower } \\
\text { BF\% compared with occasional } \\
(p<0.0001) \text { and rare }(p<0.0001) .\end{array}$ & $\uparrow$ \\
\hline
\end{tabular}


Table 1. Cont.

\begin{tabular}{|c|c|c|c|c|c|c|c|c|}
\hline $\begin{array}{l}\text { Reference } \\
\text { (Author, } \\
\text { year, } n \text { ) }\end{array}$ & Study Subjects & Methods & $\begin{array}{l}\text { Skipping Breakfast } \\
\text { Definition }\end{array}$ & $\begin{array}{l}\text { Breakfast Evaluation } \\
\text { Method }\end{array}$ & OB/OW Definition & $\begin{array}{l}\text { Prevalence of } \\
\text { Breakfast Skippers }\end{array}$ & Results & $\begin{array}{l}\text { Impact of } \\
\text { Skipping } \\
\text { Breakfast } \\
\text { on OW/OB }\end{array}$ \\
\hline $\begin{array}{l}\text { Wijtzes } 2016 \\
\text { [33] }\end{array}$ & $\begin{array}{l}\mathrm{N}=5913, \mathrm{~T} 1: 4 \\
\text { years of age T2: } \\
6 \text { years of age, } \\
\text { M and F, The } \\
\text { Netherlands }\end{array}$ & $\begin{array}{l}\text { Longitudinal, parent-report } \\
\text { questionnaires, measured } \\
\text { height and weight, percent } \\
\text { fat mass by dual-energy } \\
\text { X-ray absorptiometry (at age } \\
6 \text { years) (cross-sectional data } \\
\text { considered) }\end{array}$ & $\begin{array}{l}\text { At age } 4 \text { years: weekly } \\
\text { consumption of breakfast } \\
\text { ("never," "1-2 days per } \\
\text { week," "“-4 days per } \\
\text { week," "4-6 days per } \\
\text { week," and "every day", } \\
\text { coded as 1-5); At age 6 } \\
\text { years: the number of days } \\
\text { of breakfast consumption } \\
\text { assessed separately for } \\
\text { weekdays (coded as 0-5) } \\
\text { and weekend days (coded } \\
\text { as } 0-2 \text { ), and the scores were } \\
\text { summed to calculate total } \\
\text { weekly consumption (0-7). } \\
\text { Breakfast skipping defined } \\
\text { as consumption <7 days per } \\
\text { week }\end{array}$ & $\begin{array}{l}\text { Food frequency } \\
\text { questionnaire }\end{array}$ & $\begin{array}{l}\text { Age- and } \\
\text { sex-specific BMI } \\
\text { cut-offs according } \\
\text { to IOTF criteria }\end{array}$ & Not reported & $\begin{array}{l}\text { Breakfast skipping at age } 4 \text { years } \\
\text { associated with increased \% fat } \\
\text { mass at age } 6 \text { years }(\beta=1.38 ; \\
95 \% \text { CI: } 0.36-2.40)\end{array}$ & $\uparrow$ \\
\hline $\begin{array}{l}\text { Fayet-Moore } \\
2016 \text { [34] }\end{array}$ & $\begin{array}{l}\mathrm{N}=4487, \text { aged } \\
2-16 \text { years, } \mathrm{M} \\
\text { and } \mathrm{F}, \text { Australia }\end{array}$ & $\begin{array}{l}\text { Cross-sectional, } \\
\text { computer-assisted interview } \\
\text { based on 24-h recall } \\
\text { methodology over two days } \\
\text { from participants or their } \\
\text { caregivers, measured height } \\
\text { and weight }\end{array}$ & $\begin{array}{l}\text { Breakfast skippers were } \\
\text { children who did not } \\
\text { consume an energy } \\
\text { containing food or beverage } \\
\text { during breakfast on } 2 \text { recall } \\
\text { days }\end{array}$ & 24-h recall methodology & $\begin{array}{l}\text { BMI } z \text {-score or } \\
\text { centile adjusted for } \\
\text { age and sex was } \\
\text { calculated using the } \\
\text { US CDC } 2000 \\
\text { growth reference } \\
\text { chart }\end{array}$ & $4 \%$ & $\begin{array}{l}\text { Higher prevalence for OW/OB in } \\
\text { breakfast skippers than in } \\
\text { breakfast consumers }(21.2 \% \text { and } \\
23.2 \% \text { versus } 16.4 \% \text { and } 16.5 \% \text {, } \\
\text { respectively) }\end{array}$ & $\uparrow$ \\
\hline $\begin{array}{l}\text { Alsharairi } \\
2016 \text { [35] }\end{array}$ & $\begin{array}{l}\mathrm{T} 1 \text { (2006): } \mathrm{N}= \\
\text { 4601, 2-3 age of } \\
\text { years T2 (2008): } \\
\mathrm{N}=4381,4-5 \\
\text { years of age, } \mathrm{M} \\
\text { and F, Australia }\end{array}$ & $\begin{array}{l}\text { Cross-sectional and } \\
\text { longitudinal study, } \\
\text { face-to-face mother's } \\
\text { interview, measured height } \\
\text { and weight }\end{array}$ & $\begin{array}{l}\text { Breakfast consumption in } \\
\text { the day of interview } \\
\text { (yes/no) }\end{array}$ & Not specified & $\begin{array}{l}\text { Age- and } \\
\text { sex-specific BMI } \\
\text { cut-offs according } \\
\text { to IOTF criteria }\end{array}$ & Not reported & $\begin{array}{l}\text { OB boys at T1 (OR 2.38, } 95 \% \text { CI: } \\
1.04-5.43 \text { ) and T2 (OR 2.32, } 95 \% \text { CI: } \\
1.01-5.32 \text { ) and OB girls at T2 (OR } \\
2.26,95 \% \text { CI } 1.14-4.46 \text { ) were more } \\
\text { likelely to skip breakfast compared } \\
\text { with non-overweight }\end{array}$ & $\uparrow$ \\
\hline $\begin{array}{l}\text { Fayet-Moore } \\
2017 \text { [36] }\end{array}$ & $\begin{array}{l}\mathrm{N}=2812, \text { aged } \\
2-18 \text { years, } \mathrm{M} \\
\text { and } \mathrm{F}, \text { Australia }\end{array}$ & $\begin{array}{l}\text { Cross-sectional, face-to-face } \\
\text { interviews, measured height } \\
\text { and weight }\end{array}$ & $\begin{array}{l}\text { Breakfast skipping or eating } \\
\text { during the } 24 \mathrm{~h} \text { prior to the } \\
\text { interview day }\end{array}$ & 24-h recall methodology & $\begin{array}{l}\text { Age- and } \\
\text { sex-specific BMI } \\
\text { cut-offs according } \\
\text { to the WHO criteria }\end{array}$ & $9 \%$ & $\begin{array}{l}\text { No associations between } \\
\text { anthropometric measures and } \\
\text { breakfast or breakfast cereal choice } \\
\text { were found Breakfast skippers: } \uparrow \\
\text { higher saturated fat intake } \downarrow \\
\text { intakes of dietary fibers and most } \\
\text { micronutrients }(p<0.001)\end{array}$ & $\Leftrightarrow$ \\
\hline
\end{tabular}


Table 1. Cont.

\begin{tabular}{|c|c|c|c|c|c|c|c|c|}
\hline $\begin{array}{l}\text { Reference } \\
\text { (Author, } \\
\text { year, } n \text { ) }\end{array}$ & Study Subjects & Methods & $\begin{array}{l}\text { Skipping Breakfast } \\
\text { Definition }\end{array}$ & $\begin{array}{l}\text { Breakfast Evaluation } \\
\text { Method }\end{array}$ & OB/OW Definition & $\begin{array}{l}\text { Prevalence of } \\
\text { Breakfast Skippers }\end{array}$ & Results & $\begin{array}{l}\text { Impact of } \\
\text { Skipping } \\
\text { Breakfast } \\
\text { on OW/OB }\end{array}$ \\
\hline $\begin{array}{l}\text { Smith } 2017 \\
\text { [37] }\end{array}$ & $\begin{array}{l}\mathrm{N}=1592, \text { aged } \\
2-17 \text { years, } \mathrm{M} \\
\text { and } \mathrm{F} \text {, Australia }\end{array}$ & $\begin{array}{l}\text { Cross-sectional, } \\
\text { computer-assisted interview } \\
\text { based on } 24-\text { recall } \\
\text { methodology (for } 2-5 \text { year } \\
\text { children completed by an } \\
\text { adult; for 6-8-years an adult } \\
\text { was interviewed with help } \\
\text { from the child; } 9-11 \text { year } \\
\text { children were interviewed } \\
\text { directly with assistance from } \\
\text { an adult; } 12-17-y e a r \text { were } \\
\text { interviewed directly, with } \\
\text { the adult remaining in the } \\
\text { room for those aged } 12-14 \\
\text { years); measured weight } \\
\text { and height }\end{array}$ & $\begin{array}{l}\text { Breakfast skippers if they } \\
\text { did not define an eating } \\
\text { occasion as "breakfast' in } \\
\text { the } 24-\text {-h recall or the energy } \\
\text { intake for the "breakfast" } \\
\text { occasion was }<210 \mathrm{~kJ}\end{array}$ & 24-h recall methodology & $\begin{array}{l}\text { Age- and } \\
\text { sex-specific BMI } \\
\text { cut-offs according } \\
\text { to Cole's definition }\end{array}$ & $\begin{array}{l}11.8 \% \text { of boys and } \\
14.8 \% \text { girls skipped } \\
\text { on one day and } \\
1.4 \% \text { boys and } 3.8 \% \\
\text { girls skipped on } \\
\text { both days }\end{array}$ & $\begin{array}{l}\text { The odds of skipping breakfast } \\
\text { were progressively higher with } \\
\text { increasing BMI category }\end{array}$ & $\uparrow$ \\
\hline $\begin{array}{l}\text { Gotthelf } 2017 \\
\text { [38] }\end{array}$ & $\begin{array}{l}\mathrm{N}=2083, \text { aged } \\
9-13 \text { years, } \mathrm{M} \\
\text { and } \mathrm{F} \text {, Argentina }\end{array}$ & $\begin{array}{l}\text { Cross-sectional, self-reported } \\
\text { questionnaires compiled by } \\
\text { children and parents, } \\
\text { measured weight and height }\end{array}$ & $\begin{array}{l}\text { Breakfast habit: eating } \\
\text { breakfast on the day of the } \\
\text { survey (yes/no). Frequency: } \\
\text { always (6-7 days/week), } \\
\text { sometimes } \\
\text { (2-5 days/week), never (0-1 } \\
\text { day/week). }\end{array}$ & $\begin{array}{l}\text { Food frequency } \\
\text { questionnaire }\end{array}$ & $\begin{array}{l}\text { BMI } z \text { - scores } \\
\text { calculated based on } \\
\text { the WHO criteria }\end{array}$ & $\begin{array}{l}64.1 \% \text { of students } \\
\text { from peri-urban } \\
\text { schools and } 46.1 \% \\
\text { of students from } \\
\text { urban schools }\end{array}$ & $\begin{array}{l}\text { Among breakfast skippers, } 40.7 \% \\
\text { of the girls and } 54.7 \% \text { of the boys } \\
\text { were OW/OB. A higher } \\
\text { probability of skipping breakfast } \\
\text { was associated with obesity. }\end{array}$ & $\uparrow$ \\
\hline $\begin{array}{l}\text { Nilsen } 2017 \\
\text { [39] }\end{array}$ & $\begin{array}{l}\mathrm{N}=2620 \text {, aged } \\
7-9 \text { years, } \mathrm{M} \\
\text { and } \mathrm{F}, \text { Sweden }\end{array}$ & $\begin{array}{l}\text { Cross-sectional, proxy } \\
\text { questionnaire filled out by } \\
\text { the parents or guardians, } \\
\text { measured height and weight }\end{array}$ & $\begin{array}{l}\text { Number of eaten breakfasts } \\
\text { over a typical week (Every } \\
\text { day; most days, i.e., } \\
\text { 4-6 days a week; some days, } \\
\text { i.e., 1-3 days a week; Never) }\end{array}$ & $\begin{array}{l}\text { Food frequency } \\
\text { questionnaire }\end{array}$ & $\begin{array}{l}\text { Age- and } \\
\text { sex-specific BMI } \\
\text { cut-offs according } \\
\text { to Swedish national } \\
\text { growth reference }\end{array}$ & $4.6 \%$ & $\begin{array}{l}\text { Association between OW/OB and } \\
\text { not having breakfast every day } \\
\text { (OR 1.9 (CI 1.18-3.13)) }\end{array}$ & $\uparrow$ \\
\hline $\begin{array}{l}\text { Kesztyus } \\
2017[40]\end{array}$ & $\begin{array}{l}\mathrm{N}=1943 \text {, aged } \\
7.1 \pm 0.6 \text { years, } \\
\mathrm{M} \text { and } \mathrm{F}, \\
\text { Germany }\end{array}$ & $\begin{array}{l}\text { Cross-sectional, proxy } \\
\text { questionnaire administered } \\
\text { to the parents, measured } \\
\text { height, weight and WC }\end{array}$ & $\begin{array}{l}\text { 4-point scale, the results } \\
\text { were subsequently } \\
\text { dichotomized for analyses } \\
\text { (never, rarely versus often, } \\
\text { always) }\end{array}$ & $\begin{array}{l}\text { Food frequency } \\
\text { questionnaire }\end{array}$ & $\begin{array}{l}\text { Age- and } \\
\text { sex-specific BMI } \\
\text { cut-offs according } \\
\text { to Swedish national } \\
\text { growth reference; } \\
\text { abdominal obesity } \\
\text { as WHtR }>0.5 \text { or } \\
>0.47 \text { for girls and } \\
0.48 \text { for boys }\end{array}$ & $13.1 \%$ & 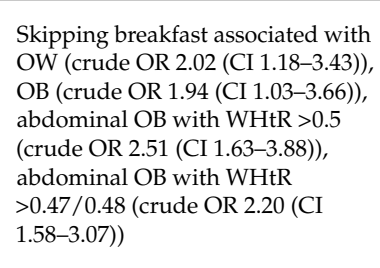 & $\uparrow$ \\
\hline $\begin{array}{l}\text { Zalewska } \\
2017 \text { [41] }\end{array}$ & $\begin{array}{l}\mathrm{N}=1999, \text { aged } \\
18 \text { years, } \mathrm{M} \text { and } \\
\mathrm{F} \text {, Poland }\end{array}$ & $\begin{array}{l}\text { Cross-sectional, self-reported } \\
\text { questionnaires, measured } \\
\text { height and weight }\end{array}$ & $\begin{array}{l}\text { Breakfast habit: skipped, }<8 \\
\text { AM, } \geq 8 \text { AM }\end{array}$ & Not specified & $\begin{array}{l}\text { BMI calculated } \\
\text { based on the WHO } \\
\text { criteria }\end{array}$ & $25 \%$ & $\begin{array}{l}\text { No difference in the prevalence of } \\
\text { breakfast skippers between NW } \\
\text { and OW/OB }\end{array}$ & $\Leftrightarrow$ \\
\hline
\end{tabular}


Table 1. Cont.

\begin{tabular}{|c|c|c|c|c|c|c|c|c|}
\hline $\begin{array}{l}\text { Reference } \\
\text { (Author, } \\
\text { year, } n \text { ) }\end{array}$ & Study Subjects & Methods & $\begin{array}{l}\text { Skipping Breakfast } \\
\text { Definition }\end{array}$ & $\begin{array}{l}\text { Breakfast Evaluation } \\
\text { Method }\end{array}$ & OB/OW Definition & $\begin{array}{l}\text { Prevalence of } \\
\text { Breakfast Skippers }\end{array}$ & Results & $\begin{array}{l}\text { Impact of } \\
\text { Skipping } \\
\text { Breakfast } \\
\text { on OW/OB }\end{array}$ \\
\hline $\begin{array}{l}\text { Coulthard } \\
2018 \text { [42] }\end{array}$ & $\begin{array}{l}\mathrm{N}=1686, \text { aged } \\
4-18 \text { years, M } \\
\text { and F, UK }\end{array}$ & $\begin{array}{l}\text { Cross-sectional, 4-day food } \\
\text { diary to be completed by the } \\
\text { children, or their parent for } \\
\text { those aged } 11 \text { years and } \\
\text { under, measured weight } \\
\text { and height }\end{array}$ & $\begin{array}{l}\text { Those consuming breakfast } \\
\text { every diary day, those } \\
\text { consuming breakfast on at } \\
\text { least one but not all diary } \\
\text { days, and those not } \\
\text { consuming breakfast on any } \\
\text { diary day }\end{array}$ & 4-day food diary & $\begin{array}{l}\text { Age- and } \\
\text { sex-specific BMI } \\
\text { cut-offs according } \\
\text { to Cole's definition } \\
\text { (1990 UK charts) }\end{array}$ & $\begin{array}{l}19.9 \% \text { of girls and } \\
14.5 \% \text { of boys }\end{array}$ & $\begin{array}{l}\text { No differences in weight status by } \\
\text { breakfast eating habits. The } \\
\text { overall nutritional profile of the } \\
\text { children in terms of fiber and } \\
\text { micronutrient intake was superior } \\
\text { in frequent breakfast consumers } \\
\text { (micronutrients: folate, calcium, } \\
\text { iron and iodine }(p<0.01)\end{array}$ & $\Leftrightarrow$ \\
\hline Tee 2018 [43] & $\begin{array}{l}\mathrm{N}=8332, \text { aged } \\
6-17 \text { years, } \mathrm{M} \\
\text { and F, Malaysia }\end{array}$ & $\begin{array}{l}\text { Cross-sectional, } \\
\text { self-administered } \\
\text { questionnaire with assistance } \\
\text { to children aged } 10 \text { years and } \\
\text { above, proxy questionnaire } \\
\text { administered to the parent } \\
\text { for children aged } 6 \text { to } 9 \text { years; } \\
\text { measured weight and height }\end{array}$ & $\begin{array}{l}\text { Breakfast skippers (ate } \\
\text { breakfast } 0-2 \text { days/week), } \\
\text { irregular breakfast eaters } \\
\text { (ate breakfast } \\
\text { 3-4 days/week) and } \\
\text { regular breakfast eaters (ate } \\
\text { breakfast } \geq 5 \text { days/week) }\end{array}$ & $\begin{array}{l}\text { Food frequency } \\
\text { questionnaire }\end{array}$ & $\begin{array}{l}\text { BMI } z \text {-scores } \\
\text { calculated based on } \\
\text { the WHO criteria }\end{array}$ & $\begin{array}{l}9.3 \% \text { in primary } \\
\text { school children and } \\
15.9 \% \text { in secondary } \\
\text { school children }\end{array}$ & $\begin{array}{l}\text { Compared to regular breakfast } \\
\text { eaters, the risk of being OW/OB } \\
\text { was higher in 6-12 years boys who } \\
\text { skipped breakfast (OR 1.71, } \\
95 \% \text { CI } 1.26-2.32) \text {, in 6-12 years } \\
\text { girls (OR 1.36, } 95 \% \text { CI } 1.02-1.81 \text { ) } \\
\text { and in } 12-17 \text { years girls (OR 1.38, } \\
95 \% \text { CI 1.01-1.90) }\end{array}$ & $\uparrow$ \\
\hline $\begin{array}{l}\text { Archero } 2018 \\
\text { [44] }\end{array}$ & $\begin{array}{l}\mathrm{N}=669, \text { aged } \\
6-16 \text { years, } \mathrm{M} \\
\text { and F, Italy }\end{array}$ & $\begin{array}{l}\text { Cross-sectional, self-reported } \\
\text { questionnaires compiled by } \\
\text { the children during } \\
\text { school-time, in the presence } \\
\text { of a teachers and medical } \\
\text { staff, measured weight } \\
\text { and height }\end{array}$ & Breakfast skipping (yes/no) & $\begin{array}{l}\text { Italian version KIDMED } \\
\text { index, a questionnaire of } \\
\text { dichotomous } \\
\text { (positive/negative) items }\end{array}$ & $\begin{array}{l}\text { Age- and } \\
\text { sex-specific BMI } \\
\text { cut-offs according } \\
\text { to IOTF criteria }\end{array}$ & $\begin{array}{l}14.8 \% \text { in primary } \\
\text { school children and } \\
21.9 \% \text { in secondary } \\
\text { school children }\end{array}$ & $\begin{array}{l}\text { OW/OB skipped breakfast more } \\
\text { frequently than NW (chi-squared } \\
3.556, p<0.04) \text {. Increased risk for } \\
\text { OW/OB in non-Italian breakfast } \\
\text { skippers (OR } 16.05, \text { CI } 95 \% \\
1.93-133.27, p<0.01 \text { ) }\end{array}$ & $\uparrow$ \\
\hline $\begin{array}{l}\text { Smith } 2010 \\
\text { [11] }\end{array}$ & $\begin{array}{l}\text { T1 (1985): } \mathrm{N}= \\
6559 ; 9-15 \text { years } \\
\text { of age T2 } \\
\text { (2004-2006): } \\
\text { 26-36 years of } \\
\text { age M and } \mathrm{F}, \\
\text { Australia }\end{array}$ & $\begin{array}{l}\text { The Childhood } \\
\text { Determinants of Adult } \\
\text { Health (CDAH) study. T1: } \\
\text { self-report questionnaires; } \\
\text { were measured: height and } \\
\text { weight. T2: self-report } \\
\text { questionnaires; were } \\
\text { measured: height, weight, } \\
\text { waist WC and BP; a venous } \\
\text { blood sample was collected } \\
\text { for lipid profile and glucose } \\
\text { metabolism }\end{array}$ & $\begin{array}{l}\text { T1: Breakfast consumption } \\
\text { was assessed by using the } \\
\text { question "Do you usually } \\
\text { eat something before } \\
\text { school?" "Yes" or "no" T2: } \\
\text { Skiping breakfast was } \\
\text { defined as not eating } \\
\text { between } 06.00 \text { and } 09.00\end{array}$ & $\begin{array}{l}\text { T1: Not specified. T2: } \\
\text { Food-frequency } \\
\text { questionnaire }\end{array}$ & $\begin{array}{l}\text { Age- and } \\
\text { sex-specific BMI } \\
\text { cut-offs according } \\
\text { to Cole's cut-off }\end{array}$ & $\begin{array}{l}\text { Skipping breakfast: } \\
14.2 \% \text { in childhood; } \\
27.5 \% \text { in adulthood }\end{array}$ & $\begin{array}{l}\text { In both childhood and adulthood: } \\
\uparrow \text { WC (mean difference: } 4.63 \mathrm{~cm} \text {; } \\
95 \% \text { CI: 1.72, } 7.53 \mathrm{~cm} \text { ) }\end{array}$ & $\uparrow$ \\
\hline
\end{tabular}


Table 1. Cont

\begin{tabular}{|c|c|c|c|c|c|c|c|c|}
\hline $\begin{array}{l}\text { Reference } \\
\text { (Author, } \\
\text { year, } n \text { ) }\end{array}$ & Study Subjects & Methods & $\begin{array}{l}\text { Skipping Breakfast } \\
\text { Definition }\end{array}$ & $\begin{array}{l}\text { Breakfast Evaluation } \\
\text { Method }\end{array}$ & OB/OW Definition & $\begin{array}{l}\text { Prevalence of } \\
\text { Breakfast Skippers }\end{array}$ & Results & $\begin{array}{l}\text { Impact of } \\
\text { Skipping } \\
\text { Breakfast } \\
\text { on OW/OB }\end{array}$ \\
\hline $\begin{array}{l}\text { Shafiee } 2013 \\
\text { [45] }\end{array}$ & $\begin{array}{l}\mathrm{N}=5625, \\
\text { subjects aged } \\
10-18 \text { years; } \mathrm{M} \\
\text { and } \mathrm{F} \text {, Iran }\end{array}$ & $\begin{array}{l}\text { The third survey of the } \\
\text { national school-based } \\
\text { surveillance system } \\
\text { (CASPIAN-III); } \\
\text { parent-report questionnaires; } \\
\text { were measured: height, } \\
\text { weight, waist WC and BP; a } \\
\text { venous blood sample was } \\
\text { collected for lipid profile and } \\
\text { glucose metabolism }\end{array}$ & $\begin{array}{l}\text { Subjects were classified into } \\
\text { three groups: "regular } \\
\text { breakfast eater" } \\
\text { (6-7 days/week), "often } \\
\text { breakfast eater"" } \\
\text { (3-5 days/week), and } \\
\text { "seldom breakfast eater" } \\
\text { (0-2 days/week) }\end{array}$ & Likert scale questionnaire & $\begin{array}{l}\text { Age- and } \\
\text { sex-specific BMI } \\
\text { cut-offs according } \\
\text { to the WHO growth } \\
\text { reference standards }\end{array}$ & $\begin{array}{l}\text { The } \% \text { of subjects } \\
\text { classified as: } \\
\text { "regular" } 47.3 \% \text {, } \\
\text { "often" } 23.7 \% \text { and } \\
\text { "seldom" } 29.0 \% \text {, } \\
\text { breakfast eaters }\end{array}$ & $\uparrow(p<0.001)$ & $\uparrow$ \\
\hline Ho 2015 [46] & $\begin{array}{l}\mathrm{N}=2401 \\
\text { elementary } \\
\text { school children; } \\
\mathrm{M} \text { and F, Taiwan }\end{array}$ & $\begin{array}{l}\text { Elementary School } \\
\text { Children's Nutrition and } \\
\text { Health Survey in Taiwan } \\
\text { (NAHSIT); self-report } \\
\text { questionnaire; were } \\
\text { measured: height, weight, } \\
\text { WC and BP; a venous blood } \\
\text { sample was collected for } \\
\text { lipid profile and glucose } \\
\text { metabolism }\end{array}$ & $\begin{array}{l}\text { Breakfast consumption was } \\
\text { assessed by using the } \\
\text { question “How often do } \\
\text { you eat breakfast in a } \\
\text { week?" The answer could } \\
\text { range from } 0 \text { to } 7 \text { times. The } \\
\text { frequency was classified } \\
\text { into three groups, including } \\
0-4,5-6 \text {, and } 7 \text { times per } \\
\text { week }\end{array}$ & $\begin{array}{l}\text { 24-h recall; } \\
\text { food-frequency } \\
\text { questionnaire The Youth } \\
\text { Healthy Eating Index for } \\
\text { the United States of } \\
\text { America (US-YHEI) } \\
\text { modified to } \\
\text { YHEI-Taiwan } \\
\text { (YHEI-TW): indicator of } \\
\text { dietary quality }\end{array}$ & Not reported & $\begin{array}{l}\% \text { Breakfast } \\
\text { frequency } \\
\text { (times/week): } 5.4 \% \\
(0-4) 5.9 \%(5-6) \\
88.7 \%(7)\end{array}$ & $\begin{array}{l}\uparrow(\text { Children who consumed } \\
\text { breakfast daily: } \\
\downarrow \text { BMI }\left(17.9 \mathrm{~kg} / \mathrm{m}^{2} ; p=0.009\right) ; \downarrow \\
\text { WC }(58.6 \mathrm{~cm} ; p=0.005))\end{array}$ & $\uparrow$ \\
\hline $\begin{array}{l}\text { Marlatt, } 2016 \\
{[47]}\end{array}$ & $\begin{array}{l}N=367, \\
\text { subjects aged } \\
11-18 \text { years; } \mathrm{M} \\
\text { and } \mathrm{F}, \\
\text { Minneapolis }\end{array}$ & $\begin{array}{l}\text { Cross-sectional study; } \\
\text { self-report survey; were } \\
\text { measured: height, weight, \% } \\
\text { body fat, and BP; a venous } \\
\text { blood sample was collected } \\
\text { for lipid profile and glucose } \\
\text { metabolism }\end{array}$ & $\begin{array}{l}\text { Breakfast consumption was } \\
\text { expressed as average } \\
\text { number of days/week that } \\
\text { breakfast was consumed }\end{array}$ & $\begin{array}{l}\text { Self-report survey using } \\
\text { validated questions } \\
\text { (Nelson MC, Lytle LA, } \\
\text { 2009. Development and } \\
\text { evaluation of a brief } \\
\text { screener to estimate } \\
\text { fast-food and beverage } \\
\text { consumption among } \\
\text { adolescents. J Am Diet } \\
\text { Assoc; 109, 730-734; 24-h } \\
\text { recalls }\end{array}$ & $\begin{array}{l}\text { Age- and } \\
\text { sex-specific BMI } \\
\text { cut-offs according } \\
\text { to the CDC Growth } \\
\text { Charts, (2000) }\end{array}$ & Not reported & $\uparrow \mathrm{BMI}$ and $\%$ body fat & $\uparrow$ \\
\hline
\end{tabular}


Table 2. Summarized studies' characteristics on metabolic variables and skipping breakfast.

\begin{tabular}{|c|c|c|c|c|c|c|c|c|c|c|c|c|}
\hline $\begin{array}{l}\text { Reference } \\
\text { (Author, } \\
\text { Year, n) }\end{array}$ & $\begin{array}{l}\text { Study } \\
\text { Subjects }\end{array}$ & Methods & $\begin{array}{l}\text { Skipping } \\
\text { Breakfast } \\
\text { Definition }\end{array}$ & $\begin{array}{l}\text { Breakfast } \\
\text { Evaluation } \\
\text { Method }\end{array}$ & OW/OB Definition & $\begin{array}{l}\text { Prevalence of } \\
\text { Breakfast } \\
\text { Skippers }\end{array}$ & $\begin{array}{l}\text { Association } \\
\text { of Skipping } \\
\text { Breakfast } \\
\text { with OW/OB }\end{array}$ & $\begin{array}{l}\text { Association of } \\
\text { Skipping Breakfast } \\
\text { with Blood } \\
\text { Pressure }\end{array}$ & $\begin{array}{l}\text { Association of } \\
\text { Skipping } \\
\text { Breakfast with } \\
\text { Lipid Profile }\end{array}$ & $\begin{array}{l}\text { Association of } \\
\text { Skipping } \\
\text { Breakfast with } \\
\text { Glucose } \\
\text { Metabolism }\end{array}$ & $\begin{array}{l}\text { Association of } \\
\text { Skipping } \\
\text { Breakfast with } \\
\text { Metabolic } \\
\text { Syndrome }\end{array}$ & $\begin{array}{l}\text { Association of } \\
\text { Skipping Breakfast } \\
\text { with Nutrient Intake }\end{array}$ \\
\hline $\begin{array}{l}\text { Smith2010 } \\
\text { [11] }\end{array}$ & $\begin{array}{l}\text { T1 }(1985): \mathrm{N}= \\
6559 ; 9-15 \\
\text { years of age. } \\
\text { T2 } \\
\text { (2004-2006): } \\
\text { 26-36 years of } \\
\text { age. M and F, } \\
\text { Australia }\end{array}$ & $\begin{array}{l}\text { The Childhood } \\
\text { Determinants of Adult } \\
\text { Health (CDAH) study. T1: } \\
\text { self-report questionnaires; } \\
\text { were measured. height and } \\
\text { weight. T2: self-report } \\
\text { questionnaires; were } \\
\text { measured: height, WC and } \\
\text { BP; a venous blood sample } \\
\text { was collected for lipid } \\
\text { profile and glucose } \\
\text { metabolism }\end{array}$ & $\begin{array}{l}\text { T1: Breakfast } \\
\text { consumption was } \\
\text { assessed by using } \\
\text { the question "Do } \\
\text { you usually yat } \\
\text { something before } \\
\text { school?" "Yef" or } \\
\text { "no". T2: Skipping } \\
\text { breakfast was } \\
\text { defined as not } \\
\text { eating between } \\
06.00 \text { and } 09.00\end{array}$ & $\begin{array}{l}\text { T1: Not specified } \\
\text { T2: } \\
\text { Food-frequency } \\
\text { questionnaire }\end{array}$ & $\begin{array}{l}\text { Age- and sex-specific } \\
\text { BMI cut-offs according } \\
\text { to Cole's cut-off }\end{array}$ & $\begin{array}{l}\text { Skipping } \\
\text { breakfast: } 14.2 \% \\
\text { in childhood; } \\
27.5 \% \text { in } \\
\text { adulthood }\end{array}$ & $\begin{array}{l}\text { In both } \\
\text { childhood } \\
\text { and } \\
\text { adulthood: } \uparrow \\
\text { WC (mean } \\
\text { difference: } \\
4.63 \mathrm{~cm} ; \\
55 \% \text { C: } 1.72, \\
7.53 \mathrm{~cm})\end{array}$ & Not reported & $\begin{array}{l}\uparrow \text { Total (mean } \\
\text { difference: } \\
0.40 \mathrm{mmol} / \mathrm{L} ; \\
95 \% \text { CI: } 0.13 \\
0.68 \mathrm{mmol} / \mathrm{L} \text { ) and } \\
\text { LDL-cholesterol } \\
\text { (mean difference: } \\
0.40 \mathrm{mmol} / \mathrm{L} ; \\
95 \% \text { CI: } 0.16 \text {; } \\
0.64 \mathrm{mmol} / \mathrm{L} \text { ) }\end{array}$ & $\begin{array}{l}\text { In both } \\
\text { childhood and } \\
\text { adulthood: } \uparrow \\
\text { fasting insulin } \\
\text { (mean difference: } \\
2.02 \mathrm{mU} / \mathrm{L} \text {; } \\
95 \% \mathrm{CI} 0.75 \\
3.29 \mathrm{mU} / \mathrm{L} \text { ) }\end{array}$ & Not reported & Not reported \\
\hline $\begin{array}{l}\text { Monzani } \\
2013[48]\end{array}$ & $\begin{array}{l}\mathrm{N}=489, \\
\text { subjects aged } \\
6.7 \text { to } 13 \text { years; } \\
\text { M and F, Italy }\end{array}$ & $\begin{array}{l}\text { Population-based, } \\
\text { cross-sectional study; } \\
\text { self-reported } \\
\text { questionnaire; were } \\
\text { measured: height, weight, } \\
\text { WC, and BP; a venous } \\
\text { blood sample was } \\
\text { collected for lipid profile, } \\
\text { uric acid and glucose } \\
\text { metabolism }\end{array}$ & $\begin{array}{l}\text { Breakfast } \\
\text { consumption: } \\
\text { yes } / \text { no }\end{array}$ & Not specified & $\begin{array}{l}\text { MetS according to } \\
\text { modified NCEP-ATP } \\
\text { III criteria of Cruz and } \\
\text { Goran }\end{array}$ & Not reported & Not reported & Not reported & Not reported & Not reported & $\begin{array}{l}\text { In school-children } \\
\text { aged } \\
\text { ag.1-13 years: no } \\
\text { breakfast } \\
\text { consumption (OR } \\
=5.0,95 \% \text { ( } 95 \\
1.5-17.2, p=0.02) \\
\text { was } \uparrow \text { in those } \\
\text { with Mets }\end{array}$ & Not reported \\
\hline $\begin{array}{l}\text { Shafiee } \\
2013 \text { [45] }\end{array}$ & $\begin{array}{l}\mathrm{N}=5625 \\
\text { subjects aged } \\
10-18 \text { years; } \\
\text { M and F, Iran }\end{array}$ & $\begin{array}{l}\text { The third survey of the } \\
\text { national school-based } \\
\text { surveillance system } \\
\text { (CASPIAN-III); } \\
\text { parent-report } \\
\text { questionnaires; were } \\
\text { measured: height, weight, } \\
\text { waist circumference (WC) } \\
\text { and blood pressure (BC); } \\
\text { venous blood sample was } \\
\text { collected for lipid profile } \\
\text { and glucose metabolism }\end{array}$ & $\begin{array}{l}\text { Subjects were } \\
\text { classified into three } \\
\text { groups: "regular } \\
\text { breaktast eater" } \\
\text { (6-7days/week), } \\
\text { "often breakfast } \\
\text { eater" } \\
\text { (3-5days/week), } \\
\text { and "seldom }) \\
\text { breakfast eater" } \\
\text { (0-2 days/week) }\end{array}$ & $\begin{array}{l}\text { Likert scale } \\
\text { questionnaire }\end{array}$ & $\begin{array}{l}\text { Age- and sex-specific } \\
\text { BMI cut-offs according } \\
\text { to the WHO growth } \\
\text { reference standards } \\
\text { Metabolic syndrome } \\
\text { (Mets) was defined } \\
\text { based on the Adult } \\
\text { Treatment Panel III } \\
\text { (ATP III) criteria } \\
\text { modified for the } \\
\text { pediatric age group }\end{array}$ & $\begin{array}{l}\text { The \% of subjects } \\
\text { classified as: } \\
\text { "regular" } 47.3 \% \text {, } \\
\text { "often" } 2.37 \% \text { and } \\
\text { "seldom" } 29.0 \% \text {, } \\
\text { breakfast eaters }\end{array}$ & $\uparrow(p<0.001)$ & $\uparrow(p<0.001)$ & $\begin{array}{l}\uparrow \text { Triglycerides, } \\
\text { LDL-cholesterol } \\
(p<0.001) \downarrow \\
\text { HDL-cholesterol }\end{array}$ & Not reported & $\begin{array}{l}\uparrow(\mathrm{O} R \mathrm{R} .96, \\
95 \% \mathrm{CI} 1.18-3.27)\end{array}$ & Not reported \\
\hline $\begin{array}{l}\text { Ho } 2015 \\
{[46]}\end{array}$ & $\begin{array}{l}\mathrm{N}=2401 \text {, } \\
\text { elementary } \\
\text { school } \\
\text { children; M } \\
\text { and F, Taiwan }\end{array}$ & $\begin{array}{l}\text { Elementary School } \\
\text { Children's Nutrition and } \\
\text { Health Survey in Taiwan } \\
\text { (NAHSIT); self-report } \\
\text { questionnaire; were } \\
\text { measured: height, weight, } \\
\text { circumference waist (WC) } \\
\text { and blood pressure (BP); a } \\
\text { venous blood sample was } \\
\text { collected for lipid profile } \\
\text { and glucose metabolism }\end{array}$ & $\begin{array}{l}\text { Breakfast } \\
\text { consumption was } \\
\text { assessed by using } \\
\text { the question "How } \\
\text { often do you eat } \\
\text { breakfast in a } \\
\text { week?" The answer } \\
\text { could range from } 0 \\
\text { to } 7 \text { times. The } \\
\text { frequency was } \\
\text { classified into three } \\
\text { groups, including } \\
0-4,5-6 \text {, and } 7 \\
\text { times per week }\end{array}$ & $\begin{array}{l}\text { 24-h recall; } \\
\text { food-frequency } \\
\text { questionnaire. } \\
\text { The Youth } \\
\text { Healthy Eating } \\
\text { Index for the } \\
\text { United States of } \\
\text { America } \\
\text { (US-YHEI) } \\
\text { modified to } \\
\text { YHEI-Taiwan } \\
\text { (YHEI-TW): } \\
\text { indicator of } \\
\text { dietary quality }\end{array}$ & $\begin{array}{l}\text { MetS was defined } \\
\text { based on criteria from } \\
\text { Cook }\end{array}$ & $\begin{array}{l}\% \text { Breakfast } \\
\text { frequency } \\
\text { (times/week): } \\
5.4 \%(0-4) 5.9 \% \\
(5-6) 88.7 \%(7)\end{array}$ & $\begin{array}{l}\uparrow(\text { Children } \\
\text { who skipped } \\
\text { breakfast } \\
\text { daily: } \mathrm{BMI} \\
\left(17.9 \mathrm{~kg} / \mathrm{m}^{2} ;\right. \\
p=0.009) ; \\
\mathrm{WC}(58.6 \mathrm{~cm} ; \\
p=0.005))\end{array}$ & 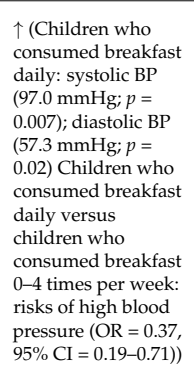 & $\begin{array}{l}\text { HDL-cholesterol } \\
\text { (Children who } \\
\text { consumed } \\
\text { breakfast daily: } \uparrow \\
\text { HDL cholesterol } \\
(59.5 \mathrm{mg} / \mathrm{dL} ; p= \\
0.03))\end{array}$ & $\Leftrightarrow$ & 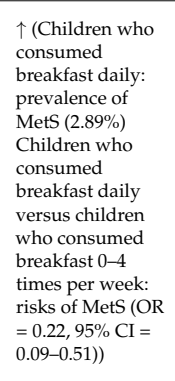 & $\begin{array}{l}\text { YHEI-TW scores } \\
\text { (Children who } \\
\text { consumed breakfast } \\
\text { daily versus those } \\
\text { who consumed } \\
\text { breakfast } 0-4 \text { times } \\
\text { per week: } \uparrow \text { intakes } \\
\text { of: saturated fat, } \\
\text { cholesterol, vitamins } \\
\text { A, B1, B2, calcium, } \\
\text { phosphorus, } \\
\text { magnesium, and } \\
\text { potassium; } \uparrow \\
\text { YHEI-TW scores } \\
\text { (better dietary } \\
\text { quality)) }\end{array}$ \\
\hline
\end{tabular}


Table 2. Cont.

\begin{tabular}{|c|c|c|c|c|c|c|c|c|c|c|c|c|}
\hline $\begin{array}{l}\text { Reference } \\
\text { (Author, } \\
\text { Year, } n)\end{array}$ & $\begin{array}{l}\text { Study } \\
\text { Subjects }\end{array}$ & Methods & $\begin{array}{l}\text { Skipping } \\
\text { Breakfast } \\
\text { Definition }\end{array}$ & $\begin{array}{l}\text { Breakfast } \\
\text { Evaluation } \\
\text { Method }\end{array}$ & OW/OB Definition & $\begin{array}{l}\text { Prevalence of } \\
\text { Breakfast } \\
\text { Skippers }\end{array}$ & $\begin{array}{l}\text { Association } \\
\text { of Skipping } \\
\text { Breakfast } \\
\text { with OW/OB }\end{array}$ & $\begin{array}{l}\text { Association of } \\
\text { Skipping Breakfast } \\
\text { with Blood } \\
\text { Pressure }\end{array}$ & $\begin{array}{l}\text { Association of } \\
\text { Skipping } \\
\text { Breakfast with } \\
\text { Lipid Profile }\end{array}$ & $\begin{array}{l}\text { Association of } \\
\text { Skipping } \\
\text { Breakfast with } \\
\text { Glucose } \\
\text { Metabolism }\end{array}$ & $\begin{array}{l}\text { Association of } \\
\text { Skipping } \\
\text { Breakfast with } \\
\text { Metabolic } \\
\text { Syndrome } \\
\end{array}$ & $\begin{array}{l}\text { Association of } \\
\text { Skipping Breakfast } \\
\text { with Nutrient Intake }\end{array}$ \\
\hline $\begin{array}{l}\text { Osawa } \\
2015 \text { [49] }\end{array}$ & $\begin{array}{l}\mathrm{N}=689 \\
\text { subjects aged } \\
10-13 \text { years; } \\
\mathrm{M} \text { and } \mathrm{F} \\
\text { Japan }\end{array}$ & $\begin{array}{l}\text { Cross-sectional study; } \\
\text { self-report questionnaire; } \\
\text { were measuredt height, } \\
\text { weight, WC and BP; } \\
\text { venous blood sample was } \\
\text { collected for lipid profile } \\
\text { and glucose metabolism }\end{array}$ & $\begin{array}{l}\text { Breakfast } \\
\text { consumption was } \\
\text { assessed by using } \\
\text { the question “Do } \\
\text { you have breakfast } \\
\text { every day? (Yes, } \\
\text { alone/Yes, with } \\
\text { family/Seldom/No) }\end{array}$ & $\begin{array}{l}\text { Food-frequency } \\
\text { questionnaire } \\
\text { designed by } \\
\text { members of the } \\
\text { Ichikawa Dental } \\
\text { Association }\end{array}$ & $\begin{array}{l}\text { MetS was defined } \\
\text { based on critieria } \\
\text { identified by the } \\
\text { Japanese society of } \\
\text { Internal Medicine, the } \\
\text { Japan Society for the } \\
\text { Study of Obesity and } \\
\text { the Ministry of Health, } \\
\text { Labour and Welfare in } \\
\text { Japan }\end{array}$ & Not reported & Not reported & Not reported & Not reported & Not reported & $\begin{array}{l}\text { Not eating } \\
\text { breakfast was } \\
\text { associated } \\
\text { significantly with } \\
\text { Mets or high risk } \\
\text { Mets (OR: 2.70, } \\
95 \% \text { CI: } 1.01-7.23 \text {, } \\
p<0.05 \text { ) }\end{array}$ & Not reported \\
\hline $\begin{array}{l}\text { Marlatt, } \\
2016 \text { [47] }\end{array}$ & $\begin{array}{l}\mathrm{N}=367 \\
\text { subjects aged } \\
11-18 \text { years; } \\
\mathrm{M} \text { and } \mathrm{F} \\
\text { Minneapolis }\end{array}$ & $\begin{array}{l}\text { Cross-sectional study; } \\
\text { self-report survey; were } \\
\text { measured height, weight, } \\
\text { BF\%, and blood pressure } \\
\text { BPF; avonous blood sample } \\
\text { was collected for lipid } \\
\text { profile and glucose } \\
\text { metabolism }\end{array}$ & $\begin{array}{l}\text { Breakfast } \\
\text { consumption was } \\
\text { expressed as } \\
\text { average number of } \\
\text { days/ week } \\
\text { breakfast was } \\
\text { consumed }\end{array}$ & $\begin{array}{l}\text { Self-report } \\
\text { survey using } \\
\text { validated } \\
\text { questions } \\
\text { (Nelson MC, } \\
\text { Lytle LA, 2009. } \\
\text { Development } \\
\text { and evaluation } \\
\text { of a brief } \\
\text { screener to } \\
\text { estimate } \\
\text { fast-food and } \\
\text { beverage } \\
\text { consumption } \\
\text { among } \\
\text { adolescents. J } \\
\text { Am Diet Assoc; } \\
\text { 109, 730-734; } \\
\text { 24-h recalls }\end{array}$ & $\begin{array}{l}\text { Age- and sex-specific } \\
\text { BMI cut-offs according } \\
\text { to the CDC Growth } \\
\text { Charts, (2000) Mets } \\
\text { was defined based on } \\
\text { the Adult Treatment } \\
\text { Panel III (ATP III) } \\
\text { criteria }\end{array}$ & Not reported & $\begin{array}{l}\uparrow \text { BMI and } \% \\
\text { body fat }\end{array}$ & $\Leftrightarrow$ & $\Leftrightarrow$ & $\uparrow$ HOMA-IR & $\begin{array}{l}\uparrow \text { MetS cluster } \\
\text { score }\end{array}$ & Not reported \\
\hline
\end{tabular}

Legend: $\mathrm{BMI}=$ Body Mass Index $\mathrm{CI}$ = Confidence Interval; F = Females; CDC = Center for Disease Control and Prevention; $\mathrm{M}=$ Males; MetS = Metabolic Syndrome; OB = Obesity; OR = Odd Ratio; OW = Overweight; $\uparrow=$ Increased; $\downarrow=$ Reduced; $\Leftrightarrow=$ Not Variation 
Study quality was independently assessed by three reviewers according to the Newcastle-Ottawa Scale for quality assessment of cohort studies and case-control studies [36]. The scales allocate stars, with a maximum of nine; the criteria were quality of selection (maximum, four stars), comparability (maximum, two stars), and exposure (maximum, three stars). High quality was assessed for more than eight stars.

\section{Results}

The literature search identified 239 potentially relevant articles. After reviewing the titles and abstracts and the full-length articles, 39 articles were selected for closer assessment and then included in our analysis [11-49]. The search flow-chart is represented in Figure 1. Agreement between reviewers on which studies to include was good: the $\mathrm{K}$ for the agreement was $85 \%$ after screening titles and abstracts and $100 \%$ after screening full-text articles. Overall, data from a total of 286,804 children and adolescents, living in 32 countries, were reported (Figure 2).
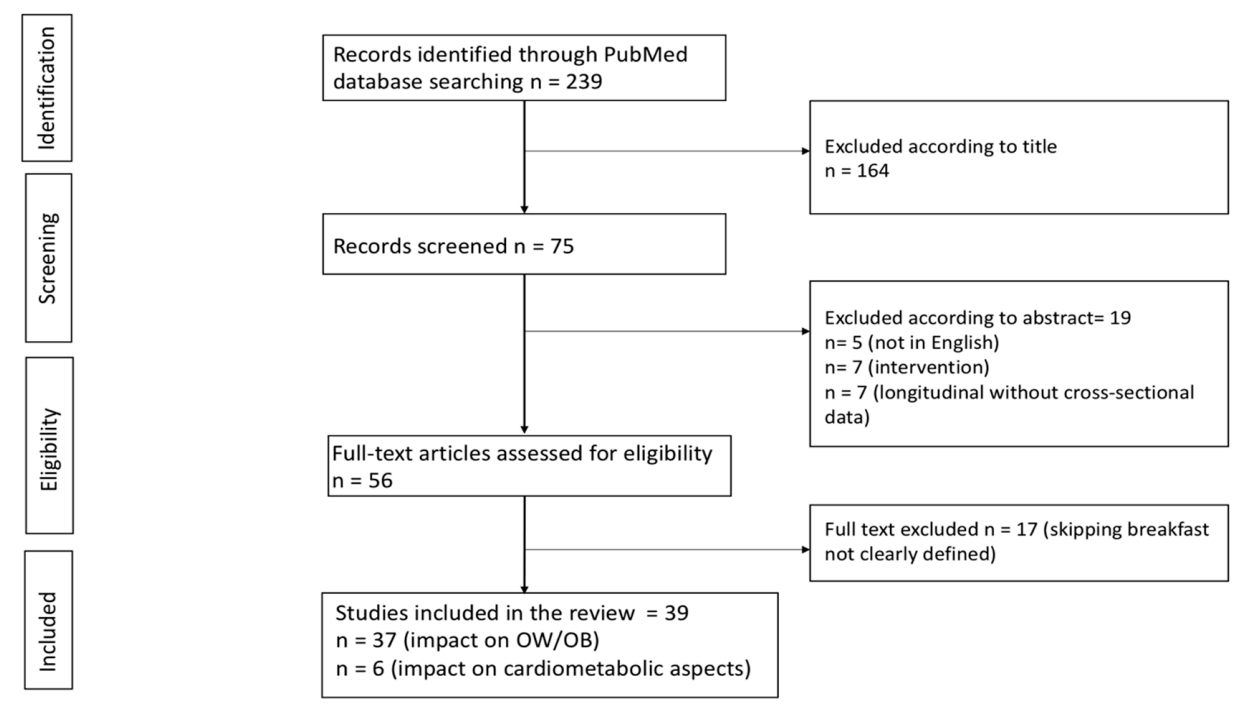

Figure 1. Flow diagram for study retrieval and selection.
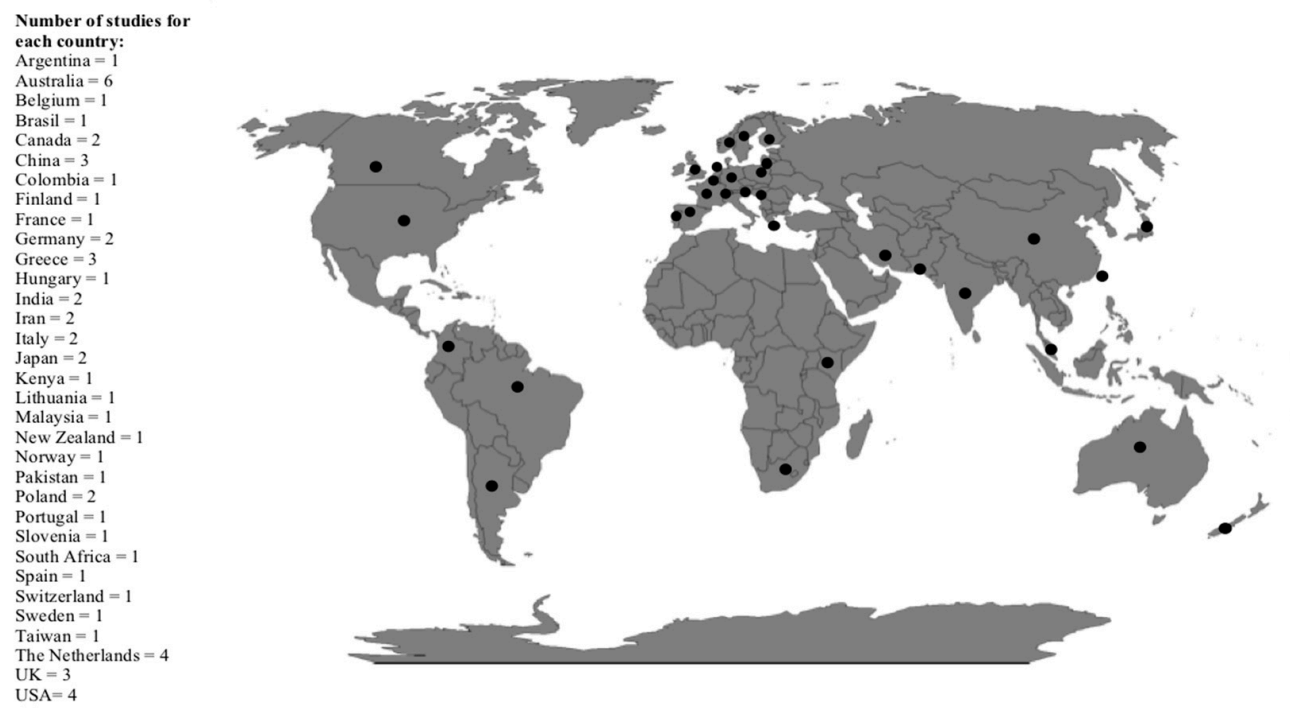

Figure 2. Countries' distribution of the 39 selected studies.

Study quality was reported in Supplemental Tables S1 and S2. The risk of bias was relatively high because (1) exclusion and inclusion criteria were not always clear; (2) some of the studies were not 
gender- and age-balanced; (3) data on subgroups were difficult to be extrapolated; (4) some studies did not clearly describe how the allocation was performed in cases and controls (in particular for the definition of skipping breakfast); (5) some of the studies could have a selective reporting bias (mis-reporting or under-reporting of breakfast habits due to methods used for the dietary assessment); (6) methods for the definition of skipping breakfast, and $\mathrm{OW} / \mathrm{OB}$ were heterogeneous or not reported; (7) methods for the evaluation of nutrition assessment were heterogeneous; (8) confounding factors were lacking or not clearly reported in most of the studies.

\subsection{Association of Skipping Breakfast with Overweight/Obesity}

Thirty-seven out of 39 articles were selected for closer assessment of weight and then included in our analysis [11-47]. They are summarized in Table 1 . Of the 37 selected papers, 32 were cross-sectional studies and 5 were longitudinal studies reporting cross-sectional data $[11,13,23,29,33]$. Overall, data from a total of 285,626 children were reported. They came from 33 different countries (Figure 2). Children's age showed a wide range of variability ranging from 44 months to 21.2 years. Only one study included adolescents older than 18 years, and, although it partly failed to respect all the inclusion criteria, we did not exclude it because it was conducted in a school population; the age range depended on the repetition of grades and the population older than 18 years was only the 5.5\% [12]. One of the studies included a sample of 9-15 years old, enrolled in 1985, with a follow-up of about 20 years (2004-2006) at ages 26-36 years [11]. One study included only preschool-aged children [13], 27 studies included only school-aged children and adolescents [1,11,14-24,26-28,31,32,38-41,43-47], and 9 studies analyzed both preschoolaged and school-aged children and adolescents [25,29,30,33-37,42]. Most studies recorded data about breakfast skipping by food frequency questionnaires [11,14,16,18,20,22,27-29,31-33,38-40,43-47], some on a recall-based methodology or by food diaries $[13,15,21,24,30,34,36,37,42,46]$, in others yes/no answers or unspecified methods were used $[11,12,17,19,23,25,26,35,41,44]$. Questionnaires were administered to the children/adolescents or to the parents in the case of youngsters.

The subjects' weight and height were measured in most cases, whereas they were reported only in one study [16]. To define overweight and obesity, BMI z-scores or age- and sex-specific cut-offs according to international criteria were used in most studies, whereas national-specific growth charts were referred to in 4 papers $[25,29,39,40]$.

The reported prevalence of breakfast skippers showed extremely wide variability, ranging from $0.7 \%$ to $74.7 \%$ according to the definition of breakfast skipping used. Seven studies did not report the prevalence of breakfast skippers $[19,25-27,33,35,47]$.

Almost all the included studies concluded that skipping breakfast is associated with an increased risk or prevalence of $\mathrm{OW} / \mathrm{OB}$, in some cases with limitations: Mustaq et al. reported that skipping breakfast was associated with overweight only among girls [24], similarly to what reported by Januszek-Trzciakowska et al. [28] with respect to obesity. Only 6 studies reported no association between anthropometric measures and the habit of skipping breakfast [25] for 3-10 years aged children $[29,36,41,42]$. Overall, a positive association between skipping breakfast and OW/OB was found in 270,362 subjects ( $94.7 \%$ ).

In most studies the crude association between body fatness measures and skipping breakfast was considered, whereas in some others adjustments were performed for potential confounding factors, i.e., sex, age, ethnicity, smoking, dieting, physical activity, and parental education [12]; gender, family situation (single-parent family), ethnic background, education level and smoking [16]; eating fast food, and physical activity and sedentary lifestyle [24].

\subsection{Association of Skipping Breakfast with Metabolic and Nutritional Aspects}

The literature search identified 34 potentially relevant articles. After reviewing the titles and abstracts and the full-length articles, 6 articles were selected for closer assessment and then included in our analysis [11,45-49]. They are summarized in Table 2. 
Of the 6 selected papers, 5 were cross-sectional studies and 1 was a longitudinal study reporting cross-sectional data [11,45-49]. Overall, data from a total of 16,130 children were reported. They came from 6 different countries. Children's age showed a wide range of variability ranging from 6 years to 18 years. The longitudinal study included a sample of 9-15 years old, enrolled in 1985, with a follow-up of about 20 years (2004-2006) at ages 26-36 years [11]. Most studies recorded data about breakfast skipping by food frequency questionnaires $[11,45-47,49]$, some on a recall-based methodology or by food diaries [46], in others yes/no answers or not specified methods were used [11,48]. Questionnaires were administered to the children/adolescents or to the parents in the case of youngsters.

In all studies, anthropometric data, such as weight, height, BMI, waist circumference, and blood pressure were collected [11,45-49], while in one study the percentage of body fat was also reported [47]. Blood samples for the evaluation of glucose metabolism and lipid profile were collected [11,45-49].

Metabolic syndrome (MetS) was described in 5 studies [45-49]. In some of the studies, MetS was defined using NCEP-ATP III criteria modified by Cruz and Goran for the pediatric age $[45,48]$ whereas in one study NCEP ATP III adult criteria [47]. Ho CY et al. used specific criteria from Cook [46], while Osawa $\mathrm{H}$ et al. those identified by national scientific societies and the Ministry of Health, Labour and Welfare in Japan [49].

One study included an indicator of dietary quality named "The Youth Healthy Eating Index for the United States of America (US-YHEI)" modified to YHEI-Taiwan [46]. YHEI-TW scores was calculated using the 24-hour dietary recall and FFQs, with higher scores indicating a better diet. The items included whole grains, vegetables, fruits, dairy, meat ratio, snack foods, sweetened beverages, multivitamins, fried foods outside the home, consumption of breakfast and, dinner with the family.

The reported prevalence of breakfast skippers had a huge variability, ranging from $5.4 \%$ to $29.0 \%$ according to the definition of breakfast skipping used. Three studies did not report the prevalence of breakfast skippers [47-49].

Only 3 of the included studies [45-47] investigated the correlation between breakfast consumption and blood pressure, with a significant negative association in 2 of them [45,46].

In 4 out of the 6 studies [11,45-47], the correlation between breakfast consumption and lipid profile was evaluated. Subjects who skipped breakfast had lower HDL-cholesterol levels [45,46], increased triglycerides [45], total and LDL-cholesterol [11,45].

Only 3 of the studies $[11,46,47]$ investigated glucose metabolism. Two of them reported a higher insulin-resistance in who skipped breakfast [11,47].

Almost all the studies concluded that skipping breakfast is associated with an increased risk or prevalence of MetS [45-49].

Ho CY et al. reported that children who consumed breakfast daily had higher intakes of saturated fat, cholesterol, vitamins A, B1, B2, calcium, phosphorus, magnesium, and potassium and better dietary quality in comparison with those who consumed breakfast $<4$ times per week. Diversely, breakfast skippers had the highest proportion (25.5\%) of under-reporting energy intake than the controls [46].

\section{Discussion}

This systematic review confirms with more recent data and strengthens the evidence summarized in 2010 [6] indicating that children and adolescents who skip breakfast are at higher risk to be or become OW/OB. Skipping breakfast seems also associated with metabolic syndrome presence, but data are still anecdotal.

The last decades are characterized by an increasing incidence of pediatric obesity, determined by the change of many lifestyle factors, including the diet. The determination of more risky dietary habits has an impact on public health to plan prevention tailored programs.

We analyzed the studies on children and adolescents published in the last ten years, not covered by the previous systematic review [6] with the purpose of analyzing trends and increasing knowledge. Unexpectedly, we failed to find in children and adolescents intervention studies or RCTs aiming to analyze the causative effect of skipping breakfast on $\mathrm{OW} / \mathrm{OB}$ and related comorbidities. 
Firstly, we observed a prevalence of breakfast skippers ranging $1.3-74.7 \%$, according to different definitions used for the skipping breakfast. However, most of the studies reported that at least $10-30 \%$ (mean \pm SD: $16.0 \pm 16.2 \%$ ) of children and adolescents did not ever eat breakfast [11,13-16,18,20-22,24,26-34,36-40,42-47]. The data are consistent because the studies covered 286,804 pediatric individuals living in Europe, the US, Australia, New Zealand, Asia, and Africa. They included also pre-school age individuals, although data on them are difficult to extrapolate $[25,29,30,33-37,42]$. On the other hand, an increasing trend in skipping breakfast from childhood to adolescence is seen [21,22,43,44], as well as higher values in girls than in boys [28,37,42]. The prevalence is almost like to results reported in 2010 [6]. This means that, although epidemiological data on pediatric dietary habits are substantial, health plans to educate at a dietary day composed of 4-5 meals have been inefficacious, in particular in adolescents, or not portrayed yet. Furthermore, no studies investigated why breakfast was skipped, whilst some hypotheses have been widely discussed in literature including a lack of time, not feeling hungry in the morning, and weight concerns [50].

Secondly, some critical issues on methods should be underlined. In fact, the definition of skipping breakfast varied among all the studies, although the previous meta-analysis had already suggested improving this methodological topic in future studies to reach more significant results [6] and, recently, the American Heart Association proposed definitions to improve and make generalizable the research on this topic [51]. Most of the studies used a dummy (yes/no) or ordinal categories, based on the number of days without having a breakfast during the week $[11,12,14-17,19,22-27,29-39,41-44,48,49]$. On the other hand, some others used qualitative and unspecific categories (usually, often etc.) $[13,18,20,21,28,40,45-47]$ making a comparison among the studies difficult. We can speculate that the metabolic balance reflects likely daily circadian rhythms influenced by prevalent meal schedules [52], but rigorous methods are needed to ascertain this hypothesis. Furthermore, the dietary history was collected with validated semi-quantitative or qualitative methods only in some studies [11,13-16,18,20-22,24,27-40,42-47,49]. The nutritional assessment is always difficult with varying degree of reliability and needs carefully handling, even more in children when parents or caregivers should be engaged. The lack of accuracy may increase the already in itself high prevalence of mis-reporting and under-reporting biases, mainly in obese individuals [53].

Despite several methodological limitations, skipping breakfast in children and adolescents is associated with OW/OB in most of the studies [12-27,30-35,37-40,43,44]. Only 6 studies did not report an association or reported it only in specific categories of subjects [24,28,29,36,41,42]. Even if these studies are comparable to the others with respect to the administration of questionnaires (self-reported completed by children or parents, or face-to-face interviews) and are not restricted to a specific country, several hypotheses could be considered. Firstly, most of them used a definition of skipping breakfast more general or referred only to the day before the study $[24,29,36,41]$. Since Coulthard JD and colleagues used the UK chart published in 1995 to define OW or OB, the cut-offs different from WHO or recent IOTF growth charts could have significantly contributed [42]. An interesting point refers to the lack of association with $\mathrm{OW} / \mathrm{OB}$ in infants compared to older children. Kupers LK et al. failed to observe a higher risk of OW/OB in children aged 2 or 5 years [29], and Kuriyan R et al. a higher waist circumference in those younger than 10 years, but the association became significant only in those older than 10 years [25]. Moreover, in 3 out of 6 studies without an association with the risk of OW/OB breakfast skippers are mainly adolescent girls older than 14 years $[36,41,42]$. Coulthard JD and colleagues observed that most of the breakfast skippers were adolescent girls and that a higher proportion of girls in the 11-18-year-old age group stated that they were currently dieting than boys [42]. Moreover, $77.6 \%$ of the cohort published by Zalewska $\mathrm{M}$ et al. was composed of girls of 18 years old beyond the dietary assessment with a non-standard questionnaire [41]. Similarly, 61\% of breakfast skippers of the studies of Fayet-Moore F et al. were again in the 14-18-year-old group [36]. On the other hand, two studies reported a higher risk of OW/OB only in females who skipped breakfast, but these populations are younger (5-12 years) [24,28] than the latter [36,41,42]. These outwardly contrasting data could be secondary to the fact that the time of puberty is characterized by the starting 
peak of OW/OB incidence and females enter before males in this period. Even so, in all, these findings suggest that in studies focusing mainly on infants or adolescents more attention to subgroups and related age-specific diseases is needed. The weight-control behaviors and puberty should be carefully considered, and this group should be analyzed separately. It is known that chronotype varies with age and environmental factors [52,54-56]. We can hypothesize that desynchronization of circadian rhythms does not still occur in infants and it is not so hitting on weight balance in infants. Conversely, in very young children the reason of skipping breakfast could vary including breastfeeding during the night, loss of appetite in a failure to thrive due to several diseases (malabsorption, sleep apnea etc.).

On the other hand, none of these studies can explain why skipping breakfast is associated with OW/OB. Some authors suggest that breakfast skipping is affected by collinearity with confounding factors such as sleep duration and quality, circadian rhythms with more eating in the evening, length of night fasting, and lower physical activity levels $[6,42,52,55,56]$. Some of the selected studies corrected for confounders $[12,16,24]$, but more tailored study designs are needed. This suggestion is strengthened if we observed that Fayet-Moore and colleagues reported an association in a study [34] and failed to confirm it in another one [36]. The authors used in both the occasions data from the National Nutrition and Physical Activity Survey, conducted in 2007 [34], and 2011-2012 [36], respectively. Because the period between the two surveys is relatively short, not captured confounders seem to be the more realistic hypothesis.

All the aspects discussed (growth charts, the definition of skipping breakfast, age-dependent responses, gender, and confounding factors) are critical issues for the planning of future intervention trials.

As already anticipated, our review is not able to clarify if the OW/OB phenotype is secondary to a higher energy intake during the following hours in children who skipped breakfast. The data in the literature on this topic are few and controversial $[6,42,52,57]$. However, in the paper we selected, it seems that children and adolescents eating breakfast have a better nutritional profile in terms of micro- and macronutrients $[13,36,42,44,46]$ and is in line with data on adolescents consuming 5 meals including breakfast [58]. How this aspect impacts on metabolism is still a matter of investigation.

Lastly, we aimed to evaluate if children and adolescents who were skippers had different metabolic phenotype. Unexpectedly, the published papers are few, counting only 6 studies, but enclosed about 16,000 children. This is surprising if we consider the long-lasting impact of $\mathrm{OW} / \mathrm{OB}$ on metabolic and cardiovascular diseases.

All the studies included reported that breakfast skippers had a worse lipid profile and/or a higher prevalence of MetS [11,45-49]. Two studies also reported higher insulin [11,47] and another higher blood pressure levels [46] than those that ate breakfast regularly. All these results mirror mournfully those in adults [51].

The findings on higher cholesterol, triglycerides, and blood pressure levels have been mainly linked to higher insulin resistance in the morning [11,51]. Regarding cholesterol, higher fasting insulin levels may trigger higher hydroxyl methyl glutaryl Co-A (HMG-CoA) reductase expression, resulting in increased circulating concentrations [11]. On the other hand, because the nutrition quality of breakfast skippers seems worse, the role of higher intake of saturated fats, simple carbohydrates and salt in the other meals is another plausible explanation.

The increasing risk of MetS is likely associated again to higher insulin-resistance in breakfast skippers, but also to increased fat oxidation and low-grade inflammation $[59,60]$. Moreover, it could be partly mediated by a higher BMI, as recently shown in a meta-analysis of prospective cohorts for the risk of type 2 diabetes in adults [61]. On the other hand, Mets is strictly associated with other unhealthy lifestyles such as poor physical activity. However, it has been demonstrated that skipping breakfast adversely modulates clock and clock-controlled gene expression resulting in increased postprandial glycemic response in both healthy individuals and individuals with diabetes and MetS [62]. Some clock genes are associated also with lipid metabolism and the development of MetS [51], making the picture more colored and intriguing.

Although findings from observational studies cannot establish causality, more prospective and intervention studies from childhood to adulthood with the assessment of clinical end-points, including 
cardiovascular and metabolic diseases could provide insight into the associations we reviewed. In adults, contrary to observational data, skipping breakfast has not been found to be causal for obesity, metabolic alterations or hungry perception under the conditions so far examined in RCTs $[57,63,64]$. RCTs are needed to test the association in children and adolescents and confirm our recommendations. Moreover, authors should pay attention to all the confounders suggested and skipping breakfast, as well as nutritional assessment, should be defined and evaluated by using definitions and tools with an international agreement, as suggested by the American Heart Association [51]. The better investigation of all these aspects could help in validating "skipping breakfast" as a marker of risk of OW/OB. The "chrono-nutrition" has been investigated within the context of obesity since studies have shown that eating at the "wrong" time of day can induce weight gain, despite a similar caloric intake [55]. In this context, the role of skipping meals, in particular, breakfast that stops the night fasting, caught the eyes of researchers. On the other hand, many other players have been still poorly investigated, and in the future could explain some of the controversial results. Among the others, also the nutrient content of the daily meals could have an impact on the desynchronization of circadian rhythms implicated in the obesity increase [52]. Moreover, food timing seems to have a higher heritability, also for breakfast [65]. The length of night fasting, the presence of time-restricted feeding, the composition of nutrients of the last meal before sleeping, the chronotype of individuals, the food timing behaviors of parents and sibling should be all investigated with respect to breakfast skipping or not in further studies to reduce residual confounders beyond sleeping habits, daily food quality and physical activity. Since high plasticity and reprogramming of most metabolic patterns in pediatric age has been described [66], filling this gap is an unmet need.

With these limitations, our study provides data supporting skipping breakfast as a potential "marker" of lifestyle behaviors (yet to be elucidated) in children and adolescents that promote OW/OB and metabolic diseases. Additional studies would be helpful to standardize the definition and assessment method for breakfast skipping in order to establish the best practice for using it as a tool for assessing obesity risk.

Supplementary Materials: The following are available online at http:/ / www.mdpi.com/2072-6643/11/2/387/s1, Table S1: Newcastle-Ottawa Quality Assessment Scale for studies on weight and skipping breakfast, Table S2: Newcastle-Ottawa Quality Assessment Scale for studies on metabolic variables and skipping breakfast.

Author Contributions: Conceptualization, F.P. and S.B.; Methodology, A.M., R.R., M.C., F.P, Software, A.S.; Validation, M.C., F.A., Formal Analysis, A.M., R.R., M.C., F.P.; Investigation, A.M., R.R., M.C., F.A., A.S., Data Curation, F.A.; Writing-Original Draft Preparation, A.M., R.R., M.C., F.P.; Writing-Review and Editing, S.B.; Supervision, S.B., F.P.; Funding Acquisition, F.P.

Funding: This research received no external funding.

Acknowledgments: The study was supported by the Department of Health Sciences, University of Piemonte Orientale. The funder had no role in the study design, data collection and analysis. The Authors should thank Alessandro Bisceglia for the technical advices.

Conflicts of Interest: The authors declare no conflict of interest.

\section{References}

1. Ng, M.; Fleming, T.; Robinson, M.; Thomson, B.; Graetz, N.; Margono, C.; Mullany, E.C.; Biryukov, S.; Abbafati, C.; Abera, S.F.; et al. Global, regional, and national prevalence of overweight and obesity in children and adults during 1980-2013: A systematic analysis for the Global Burden of Disease Study 2013. Lancet 2014, 384, 766-781. [CrossRef]

2. Moraeus, L.; Lissner, L.; Yngve, A.; Poortvliet, E.; Al-Ansari, U.; Sjöberg, A. Multi-level influences on childhood obesity in Sweden: Societal factors, parental determinants and child/'s lifestyle. Int. J. Obes. 2012, 36, 969-976. [CrossRef] [PubMed]

3. Wijnhoven, T.M.A.; van Raaij, J.M.A.; Yngve, A.; Sjöberg, A.; Kunešová, M.; Duleva, V.; Petrauskiene, A.; Rito, A.I.; Breda, J. WHO European Childhood Obesity Surveillance Initiative: Health-risk behaviours on nutrition and physical activity in 6-9-year-old schoolchildren. Public. Health. Nutr. 2015, 18, 3108-3124. [CrossRef] [PubMed] 
4. Rampersaud, G.C.; Pereira, M.A.; Girard, B.L.; Adams, J.; Metzl, J.D. Breakfast habits, nutritional status, body weight, and academic performance in children and adolescents. J. Am. Diet. Assoc. 2005, 105, 743-760. [CrossRef] [PubMed]

5. Delva, J.; Omalley, P.M.; Johnston, L.D. Racial/ethnic and socioeconomic status differences in overweight and health-related behaviors among American students: National trends 1986-2003. J. Adolesc. Health. 2006, 39, 536-545. [CrossRef] [PubMed]

6. Szajewska, H.; Ruszczynski, M. Systematic review demonstrating that breakfast consumption influences body weight outcomes in children and adolescents in Europe. Crit. Rev. Food Sci. Nutr. 2010, 50, 113-119. [CrossRef]

7. Affenito, S.G.; Thompson, D.; Dorazio, A.; Albertson, A.M.; Loew, A.; Holschuh, N.M. Ready-to-eat cereal consumption and the School Breakfast Program: Relationship to nutrient intake and weight. J. Sch. Health. 2013, 83, 28-35. [CrossRef]

8. Ask, A.S.; Hernes, S.; Aarek, I.; Johannessen, G.; Haugen, M. Changes in dietary pattern in 15 years old adolescents following a 4 month dietary intervention with school breakfast—a pilot study. Nutr. J. 2006, 5, 33. [CrossRef]

9. Utter, J.; Scragg, R.; Schaaf, D.; Fitzgerald, E.; Wilson, N. Correlates of body mass index among a nationally representative sample of New Zealand children. Int. J. Pediatr. Obes. 2007, 2, 104-113. [CrossRef]

10. Vereecken, C.; Dupuy, M.; Rasmussen, M.; Kelly, C.; Nansel, T.R.; Al Sabbah, H.; Baldassari, D.; Jordan, M.D.; Maes, L.; Niclasen, B.V.; et al. Breakfast consumption and its socio-demographic and lifestyle correlates in schoolchildren in 41 countries participating in the HBSC study. Int. J. Public Health 2009, 54, 180-190. [CrossRef]

11. Smith, K.J.; Gall, S.L.; McNaughton, S.A.; Blizzard, L.; Dwyer, T.; Venn, A.J. Skipping breakfast: Longitudinal associations with cardiometabolic risk factors in the Childhood Determinants of Adult Health Study. Am. J. Clin. Nutr. 2010, 92, 1316-1325. [CrossRef] [PubMed]

12. Dialektakou, K.D.; Vranas, P.B. Breakfast skipping and body mass index among adolescents in Greece: Whether an association exists depends on how breakfast skipping is defined. J. Am. Diet. Assoc. 2008, 108, 1517-1525. [CrossRef] [PubMed]

13. Dubois, L.; Girard, M.; Kent, M.P.; Farmer, A.; Tatone-Tokuda, F. Breakfast skipping is associated with differences in meal patterns, macronutrient intakes and overweight among pre-school children. Public Health Nutr. 2008, 12, 19-28. [CrossRef] [PubMed]

14. Harding, S.; Teyhan, A.; Maynard, M.J.; Cruickshank, J.K. Ethnic differences in overweight and obesity in early adolescence in the MRC DASH study: The role of adolescent and parental lifestyle. Int. J. Epidemiol. 2008, 37, 162-172. [CrossRef] [PubMed]

15. Duncan, J.S.; Schofield, G.; Duncan, E.K.; Rush, E.C. Risk factors for excess body fatness in New Zealand children. Asia Pac. J. Clin. Nutr. 2008, 17, 138-147. [PubMed]

16. Croezen, S.; Visscher, T.L.; Ter Bogt, N.C.; Veling, M.L.; Haveman-Nies, A. Skipping breakfast, alcohol consumption and physical inactivity as risk factors for overweight and obesity in adolescents: Results of the E-MOVO project. Eur. J. Clin. Nutr. 2009, 63, 405-412. [CrossRef]

17. Nagel, G.; Wabitsch, M.; Galm, C.; Berg, S.; Brandstetter, S.; Fritz, M.; Klenk, J.; Peter, R.; Prokopchuk, D.; Steiner, R.; et al. Determinants of obesity in the Ulm Research on Metabolism, Exercise and Lifestyle in Children (URMEL-ICE). Eur. J. Pediatr. 2009, 168, 1259-1267. [CrossRef]

18. Sun, Y.; Sekine, M.; Kagamimori, S. Lifestyle and overweight among Japanese adolescents: The Toyama Birth Cohort Study. J. Epidemiol. 2009, 19, 303-310. [CrossRef]

19. Maddah, M.; Nikooyeh, B. Factors associated with overweight in children in Rasht, Iran: Gender, maternal education, skipping breakfast and parental obesity. Public Health Nutr. 2010, 13, 196-200. [CrossRef]

20. Isacco, L.; Lazaar, N.; Ratel, S.; Thivel, D.; Aucouturier, J.; Doré, E.; Meyer, M.; Duché, P. The impact of eating habits on anthropometric characteristics in French primary school children. Child Care Health Dev. 2010, 36, 835-842. [CrossRef]

21. Deshmukh-Taskar, P.; Nicklas, T.A.; Radcliffe, J.D.; O’Neil, C.E.; Liu, Y. The relationship of breakfast skipping and type of breakfast consumed with overweight/obesity, abdominal obesity, other cardiometabolic risk factors and the metabolic syndrome in young adults. The National Health and Nutrition Examination Survey (NHANES): 1999-2006. Public Health Nutr. 2013, 16, 2073-2082. [CrossRef] [PubMed] 
22. So, H.K.; Nelson, E.A.; Li, A.M.; Guldan, G.S.; Yin, J.; Ng, P.C.; Sung, R.Y. Breakfast frequency inversely associated with BMI and body fatness in Hong Kong Chinese children aged 9-18 years. Br. J. Nutr. 2011, 106, 742-751. [CrossRef] [PubMed]

23. Tin, S.P.; Ho, S.Y.; Mak, K.H.; Wan, K.L.; Lam, T.H. Breakfast skipping and change in body mass index in young children. Int. J. Obes. 2011, 35, 899-906. [CrossRef] [PubMed]

24. Mushtaq, M.U.; Gull, S.; Mushtaq, K.; Shahid, U.; Shad, M.A.; Akram, J. Dietary behaviors, physical activity and sedentary lifestyle associated with overweight and obesity, and their socio-demographic correlates, among Pakistani primary school children. Int. J. Behav. Nutr. Phys. Act. 2011, 8, 130. [CrossRef] [PubMed]

25. Kuriyan, R.; Tinku, T.; Sumithra, S.; Lokesh, D.P.; Sheth, N.R.; Joy, R.; Bhat, S.; Kurbad, A.V. Potential Factors Related to Waist Circumference in Urban South Indian Children. Indian Pediatrics. 2012, 49, 16. [CrossRef]

26. Kyeariazis, I.; Rekleiti, M.; Saridi, M.; Beliotis, E.; Toska, A.; Souliotis, K.; Wozniak, G. Prevalence of obesity in children aged 6-12 years in Greece: Nutritional behaviour and physical activity. Arch. Med. Sci. 2012, 8, 859-864. [CrossRef]

27. Van Lippevelde, W.; Te Velde, S.J.; Verloigne, M.; Van Stralen, M.M.; De Bourdeaudhuij, I.; Manios, Y.; Bere, E.; Vik, F.N.; Jan, N.; Fernández Alvira, J.M.; et al. Associations between family-related factors, breakfast consumption and BMI among 10- to 12-year-old European children: The cross-sectional ENERGY-study. PLoS One 2013, 8, e79550. [CrossRef]

28. Januszek-Trzciąkowska, A.; Małecka-Tendera, E.; Klimek, K.; Matusik, P. Obesity risk factors in a representative group of Polish prepubertal children. Arch. Med. Sci. 2014, 10, 880-885. [CrossRef]

29. Kupers, L.K.; de Pijper, J.J.; Sauer, P.J.J.; Stolk, R.P.; Corpeleijn, E. Skipping breakfast and overweight in 2and 5-year-old Dutch children-The GECKO Drenthe cohort. Int. J. Obes. 2014, 38, 569-571. [CrossRef]

30. O'Neil, C.E.; Nicklas, T.A.; Fulgoni, V.L. Nutrient intake, diet quality, and weight measures in breakfast patterns consumed by children compared with breakfast skippers: NHANES 2001-2008. AIMS Public Health. 2015, 2, 441-468. [CrossRef]

31. Smetanina, N.; Albaviciute, E.; Babinska, V.; Karinauskiene, L.; Albertsson-Wikland, K.; Petrauskiene, A.; Verkauskiene, R. Prevalence of overweight/obesity in relation to dietary habits and lifestyle among 7-17 years old children and adolescents in Lithuania. BMC Public Health. 2015, 15, 1001. [CrossRef] [PubMed]

32. Zakrzewski, J.K.; Gillison, F.B.; Cumming, S.; Church, T.S.; Katzmarzyk, P.T.; Broyles, S.T.; Champagne, C.M.; Chaput, J.P.; Denstel, K.D.; Fogelholm, M.; et al. Associations between breakfast frequency and adiposity indicators in children from 12 countries. Int. J. Obes. 2015, 5, S80-S88. [CrossRef] [PubMed]

33. Wijtzes, A.I.; Jansen, W.; Bouthoorn, S.H.; van Lenthe, F.J.; Franco, O.H.; Hofman, A.; Jaddoe, V.W.V.; Raat, H. Meal-skipping behaviors and body fat in 6-year-old children. J. Pediatr. 2016, 168, 118-125. [CrossRef] [PubMed]

34. Fayet-Moore, F.; Kim, J.; Sritharan, N.; Petocz, P. Impact of Breakfast Skipping and Breakfast Choice on the Nutrient Intake and Body Mass Index of Australian Children. Nutrients 2016, 8, 487. [CrossRef] [PubMed]

35. Alsharairi, N.A.; Somerset, S.M. Skipping breakfast in early childhood and its associations with maternal and child BMI: A study of 2-5-year-old Australian children. Eur. J. Clin. Nutr. 2016, 70, 450-455. [CrossRef]

36. Fayet-Moore, F.; McConnell, A.; Tuck, K.; Petocz, P. Breakfast and breakfast cereal choice and its impact on nutrient and sugar intakes and anthropometric measures among a nationally representative sample of australian children and adolescents. Nutrients 2017, 9, 1045. [CrossRef] [PubMed]

37. Smith, K.J.; Breslin, M.C.; McNaughton, S.A.; Gall, S.L.; Blizzard, L.; Venn, A.J. Skipping breakfast among Australian children and adolescents; findings from the 2011-2012 National Nutrition and Physical Activity Survey. Aust. N. Z. J. Public Health 2017, 41, 572-578. [CrossRef]

38. Gotthelf, S.J.; Tempestti, C.P. Breakfast, nutritional status, and socioeconomic outcome measures among primary school students from the City of Salta: A cross-sectional study. Arch. Argent. Pediatr. 2017, 115, 424-431. [CrossRef]

39. Nilsen, B.B.; Yngve, A.; Monteagudo, C.; Tellström, R.; Scander, H.; Werner, B. Reported habitual intake of breakfast and selected foods in relation to overweight status among seven- to nine-year-old Swedish children. Scand. J. Public Health 2017, 45, 886-894. [CrossRef]

40. Kesztyüs, D.; Traub, M.; Lauer, R.; Kesztyüs, T.; Steinacker, J.M. Skipping breakfast is detrimental for primary school children: Cross-sectional analysis of determinants for targeted prevention. BMC Public Health 2017, 17, 258. [CrossRef] 
41. Zalewska, M.; Maciorkowska, E. Selected nutritional habits of teenagers associated with overweight and obesity. Peer. J. 2017, 5, e3681. [CrossRef] [PubMed]

42. Coulthard, J.D.; Palla, L.; Pot, G.K. Breakfast consumption and nutrient intakes in 4-18-year-olds: UK National Diet and Nutrition Survey Rolling Programme (2008-2012). Br. J. Nutr. 2017, 118, 280-290. [CrossRef] [PubMed]

43. Tee, E.S.; Nurliyana, A.R.; Norimah, A.K.; Mohamed, H.J.B.J.; Tan, S.Y.; Appukutty, M.; Hopkins, S.; Thielecke, F.; Ong, M.K.; Ning, C.; et al. Breakfast consumption among Malaysian primary and secondary school children and relationship with body weight status - Findings from the MyBreakfast Study. Asia Pac. J. Clin. Nutr. 2018, 27, 421-432. [CrossRef] [PubMed]

44. Archero, F.; Ricotti, R.; Solito, A.; Carrera, D.; Civello, F.; Di Bella, R.; Bellone, S.; Prodam, F. Adherence to the mediterranean diet among school children and adolescents living in northern Italy and unhealthy food behaviors associated to overweight. Nutrients 2018, 10, 1322. [CrossRef] [PubMed]

45. Shafiee, G.; Kelishadi, R.; Qorbani, M.; Motlagh, M.E.; Taheri, M.; Ardalan, G.; Taslimi, M.; Poursafa, P.; Heshmat, R.; Larijani, B. Association of breakfast intake with cardiometabolic risk factors. Pediatr. 2013, 89, 575-582. [CrossRef] [PubMed]

46. Ho, C.Y.; Huang, Y.C.; Lo, W.T.C.; Wahlqvist, M.L.; Lee, M.S. Breakfast is associated with the metabolic syndrome and school performance among Taiwanese children. Res. Dev. Disabil. 2015, 44, 179-188. [CrossRef] [PubMed]

47. Marlatt, K.L.; Farbakhsh, K.; Dengel, D.R.; Lytle, L.A. Breakfast and fast food consumption are associated with selected biomarkers in adolescents. Prev. Med. Rep. 2016, 3, 49-52. [CrossRef]

48. Monzani, A.; Rapa, A.; Fuiano, N.; Diddi, G.; Prodam, F.; Bellone, S.; Bona, G. Metabolic syndrome is strictly associated with parental obesity beginning from childhood. Clin. Endoc. 2013, 81, 45-51. [CrossRef]

49. Osawa, H.; Sugihara, N.; Ukiya, T.; Ishizuka, Y.; Birkhed, D.; Hasegawa, M.; Matsukubo, T. Metabolic syndrome, lifestyle, and dental caries in japanese school children. Bull. Tokyo Dent. Coll. 2015, 56, $233-241$. [CrossRef]

50. Reddan, J.; Wahlstrom, K.; Reicks, M. Children's perceived benefits and barriers in relation to eating breakfast in schools with or without Universal School Breakfast. J. Nutr. Educ. Behav. 2002, 34, 47-52. [CrossRef]

51. St-Onge, M.P.; Ard, J.; Baskin, M.L.; Chiuve, S.E.; Johnson, H.M.; Kris-Etherton, P.; Varady, K.; American Heart Association Obesity Committee of the Council on Lifestyle and Cardiometabolic Health; Council on Cardiovascular Disease in the Young; Council on Clinical Cardiology; Stroke Council. Meal Timing and Frequency: Implications for Cardiovascular Disease Prevention: A Scientific Statement From the American Heart Association. Circulation 2017, 135, e96-e121. [CrossRef] [PubMed]

52. Oosterman, J.E.; Kalsbeek, A.; la Fleur, S.E.; Belsham, D.D. Impact of nutrients on circadian rhythmicity. Am. J. Physiol. Regul. Integr. Comp. Physiol. 2015, 308, R337-R350. [CrossRef]

53. Walker, J.L.; Ardouin, S.; Burrows, T. The validity of dietary assessment methods to accurately measure energy intake in children and adolescents who are overweight or obese: A systematic review. Eur. J. Clin. Nutr. 2018, 72, 185-197. [CrossRef] [PubMed]

54. Fleig, D.; Randler, C. Association between chronotype and diet in adolescents based on food logs. Eat. Behav. 2009, 10, 115-118. [CrossRef] [PubMed]

55. Broussard, J.L.; Van Cauter, E. Disturbances of sleep and circadian rhythms: Novel risk factors for obesity. Curr. Opin. Endocrinol. Diabetes Obes. 2016, 23, 353-359. [CrossRef] [PubMed]

56. Roßbach, S.; Diederichs, T.; Nöthlings, U.; Buyken, A.E.; Alexy, U. Relevance of chronotype for eating patterns in adolescents. Chronobiol. Int. 2018, 35, 336-347. [CrossRef] [PubMed]

57. Dhurandhar, E.J. True, true, unrelated? A review of recent evidence for a causal influence of breakfast on obesity. Curr. Opin. Endocrinol. Diabetes Obes. 2016, 23, 384-388. [CrossRef] [PubMed]

58. Jääskeläinen, A.; Schwab, U.; Kolehmainen, M.; Pirkola, J.; Järvelin, M.R.; Laitinen, J. Associations of meal frequency and breakfast with obesity and metabolic syndrome traits in adolescents of Northern Finland Birth Cohort 1986. Nutr. Metab. Cardiovasc. Dis. 2013, 23, 1002-1009. [CrossRef] [PubMed]

59. Wennberg, M.; Gustafsson, P.E.; Wennberg, P.; Hammarström, A. Poor breakfast habits in adolescence predict the metabolic syndrome in adulthood. Public Health Nutr. 2015, 18, 122-129. [CrossRef]

60. Nas, A.; Mirza, N.; Hägele, F.; Kahlhöfer, J.; Keller, J.; Rising, R.; Kufer, T.A.; Bosy-Westphal, A. Impact of breakfast skipping compared with dinner skipping on regulation of energy balance and metabolic risk. Am. J. Clin. Nutr. 2017, 105, 1351-1361. [CrossRef] 
61. Ballon, A.; Neuenschwander, M.; Schlesinger, S. Breakfast skipping is associated with increased risk of type 2 diabetes among adults: A systematic review and meta-analysis of prospective cohort studies. J. Nutr. 2018, 149, 106-113. [CrossRef] [PubMed]

62. Jakubowicz, D.; Wainstein, J.; Landau, Z.; Raz, I.; Ahren, B.; Chapnik, N.; Ganz, T.; Menaged, M.; Barnea, M.; Bar-Dayan, Y.; et al. Influences of Breakfast on Clock Gene Expression and Postprandial Glycemia in Healthy Individuals and Individuals With Diabetes: A Randomized Clinical Trial. Diabetes Care 2017, 40, 1573-1579. [CrossRef] [PubMed]

63. Dhurandhar, E.J.; Dawson, J.; Alcorn, A.; Larsen, L.H.; Thomas, E.A.; Cardel, M.; Bourland, A.C.; Astrup, A.; St-Onge, M.P.; Hill, J.O.; et al. The effectiveness of breakfast recommendations on weight loss: A randomized controlled trial. Am. J. Clin. Nutr. 2014, 100, 507-513. [CrossRef] [PubMed]

64. Sievert, K.; Hussain, S.M.; Page, M.J.; Wang, Y.; Hughes, H.J.; Malek, M.; Cicuttini, M.F. Effect of breakfast on weight and energy intake: Systematic review and meta-analysis of randomised controlled trials. BMJ 2019, 364, 142. [CrossRef] [PubMed]

65. Lopez-Minguez, J.; Dashti, H.S.; Madrid-Valero, J.J.; Madrid, J.A.; Saxena, R.; Scheer, F.A.; Ordoñana, J.R.; Garaulet, M. Heritability of the timing of food intake. Clin. Nutr. 2018. [CrossRef] [PubMed]

66. Mathias, P.C.; Elmhiri, G.; de Oliveira, J.C.; Delayeare-Orthez, C.; Barella, L.F.; Tófolo, L.P.; Fabricio, G.S.; Chango, A.; Abdennebi-Najar, L. Maternal diet, bioactive molecules, and exercising as reprogramming tools of metabolic programming. Eur. J. Nutr. 2014, 53, 711-722. [CrossRef] [PubMed]

(C) 2019 by the authors. Licensee MDPI, Basel, Switzerland. This article is an open access article distributed under the terms and conditions of the Creative Commons Attribution (CC BY) license (http://creativecommons.org/licenses/by/4.0/). 